\title{
MATHEMATICAL MORPHOLOGY: A MODERN APPROACH IN IMAGE PROCESSING BASED ON ALGEBRA AND GEOMETRY*
}

\author{
HENK J. A. M. HEIJMANS ${ }^{\dagger}$
}

\begin{abstract}
Mathematical morphology is a theory of image transformations and image functionals which is based on set-theoretical, geometrical, and topological concepts. The methodology is particularly useful for the analysis of the geometrical structure in an image. The main goal of this paper is to give an impression of the underlying philosophy and the mathematical theories which are relevant to this field. The following topics are discussed: introduction to mathematical morphology; generalization to complete lattices; morphological filters and their construction by iteration; geometrical aspects of morphology (e.g., convexity, distance, geodesic operators, granulometries, metric dilations, distance transform, cost functions); and extension of binary operators to grey-scale images.
\end{abstract}

Key words. image analysis, mathematical morphology, hit-or-miss operator. Minkowski sum and difference, dilation, erosion, opening, closing, Boolean function, complete lattice, adjunction, morphological filter, iteration of morphological operators, $\downarrow-$ (respectively, $\uparrow$-) continuous operator, metric, gauge function, convex set, geodesic operator, granulometry, metric dilation, distance transform, cost function, grey-scale morphology, flat function operator

AMS subject classifications. 69K52, 06B35, 51 K99, 52A22

1. Introduction. In the early sixties two researchers at the Paris School of Mines in Fontainebleau, Georges Matheron and Jean Serra, worked on a number of problems in mineralogy and petrography. Their main goal was to characterize physical properties of certain materials, e.g., the permeability of a porous medium, by examining their geometrical structure. Their investigations ultimately led to a new quantitative approach in image analysis, nowadays known as mathematical morphology.

Thirty years later, mathematical morphology has achieved a status as a powerful method for image processing which, besides having been applied successfully in various disciplines such as mineralogy, medical diagnostics, and histology, has also become a solid mathematical theory leaning on concepts from algebra, topology, integral geometry, and stochastic geometry. To a large extent the current status is due to its founders Matheron and Serra [28], [40], [41].

The central idea of mathematical morphology is to examine the geometrical structure of an image by matching it with small patterns at various locations in the image. By varying the size and the shape of the matching patterns, called structuring elements, one can extract useful information about the shape of the different parts of the image and their interrelations. In general the procedure results in nonlinear image operators which are well suited for the analysis of the geometrical and topological structure of an image.

Originally, mathematical morphology was developed for binary images; these can be represented mathematically as sets. The corresponding morphological operators use essentially three ingredients from set theory, namely set intersection, union, complementation and also translation. But from the very beginning there was a need for a more general theory including other object spaces such as the closed subsets of a topological space, the convex sets of a (topological) vector space, as well as spaces of functions modelling grey- scale images. Serra and Matheron [41] were the first to observe that a general theory on morphological image analysis should include the assumption that the underlying image space is a complete lattice.

This paper intends to give the reader a flavor of mathematical morphology. As such we do not aim for completeness. In fact the paper is rather fragmentary and restricts attention to those subjects which we consider to be of interest to a general mathematical readership.

* Received by the editors August 10,1992; accepted for publication (in revised form) March 17, 1994. This work is a revised and extended version of Mathematical Morphology: A Geometrical Approach in Image Processing, Nieuw Archief voor Wiskunde (Vierde Serie), Deel 10, 1992, pp. 237-276.

${ }^{\dagger}$ Centre for Mathematics and Computer Science, P.O. Box 94079, 1090 GB Amsterdam, the Netherlands. 
In the final section we point out a few other subjects which are not discussed in this paper but which may also be of interest. A general account on mathematical morophology can be found in the two books by Serra [40], [41] and in a monograph by Matheron [28]; the latter contains a comprehensive discussion on random sets and integral geometry. Actually, it is this probabilistic branch which has made morphology into such a powerful methodology, and it is somewhat unfortunate that this aspect has been given so little attention in the recent literature. Furthermore, the interested reader may refer to a monograph by Heijmans [17] dealing with various mathematical aspects of morphology. Some other elementary references are the books by Dougherty and Giardina [6], [7] and the tutorial paper by Haralick, Sternberg, and Zhuang [10].

Besides this introduction this paper comprises six sections, dealing with various aspects. Section 2 acquaints the reader with the morphological approach in image processing and discusses some basic morphological operators for binary images which are invariant under translations. In $\S 3$ we discuss an extension to the framework of complete lattices. Such an abstract theory also allows the construction of operators which are invariant under transformation groups other than translations. Section 4 deals with morphological filters; these are operators which preserve the partial ordering structure and which are idempotent. We explain how to construct filters by iteration of operators which are not idempotent but do have certain continuity properties. Geometrical aspects of morphology are discussed in $\S 5$. Despite the patchy contents of that section we hope it gives the reader an intuition for the kind of problems which emerge. Then, in $\S 6$ we discuss some extensions of the binary theory to grey-scale images, and finally, in $\S 7$, we mention some problems which have not found a place elsewhere in this paper.

\section{What is mathematical morphology?}

2.1. Hit-or-miss operator. A convenient way to model binary (=black and white) images, both continuous and discrete, is by means of sets. Unless stated otherwise we assume that $E=\mathbb{R}^{d}$ or $\mathbb{Z}^{d}$. By $\mathcal{P}(E)$ we denote the power set of $E$. The key principle underlying mathematical morphology is to gain topological or geometrical information about a binary image $X \subseteq E$ by probing it with another small set $A$, called a structuring element, at every position $h \in E$. By "probing" we mean testing whether the set $A_{h}$ hits $X$ (i.e., $A_{h} \cap X \neq \varnothing$ ), misses $X$ (i.e., $A_{h} \cap X=\varnothing$ ), or lies entirely inside $X$ (i.e., $A_{h} \subseteq X$ ), Here $A_{h}$ denotes the translate of $A$ along the vector $h: A_{h}=\{a+h \mid a \in A\}$. The hit-or-miss operator is a mapping on the space of binary images $\mathcal{P}(E)$ which is based on this intuitive idea. Let $A, B \subseteq E$ be two structuring elements such that $A \cap B=\varnothing$, and define

$$
X \circledast(A, B)=\left\{h \in E \mid A_{h} \subseteq X \text { and } B_{h} \subseteq X^{c}\right\} .
$$

Here $X^{c}$ denotes the complement of $X$, or, in image processing terminology, the background of the image $X$. See Fig. 1 for an example. It is obvious that $A$ and $B$ must have an empty intersection in order to obtain nontrivial results. If not, the resulting set will be empty.

The hit-or-miss operator is an easy example of a set operator (i.e., a mapping $\psi: \mathcal{P}(E) \rightarrow$ $\mathcal{P}(E)$ ) which is translation invariant, that is,

$$
\psi\left(X_{h}\right)=[\psi(X)]_{h} \quad \text { for } X \in \mathcal{P}(E) \text { and } h \in E .
$$

Moreover, Banon and Barrera [1] have shown that every translation invariant set operator can be represented as a union of hit-or-miss operators.

Proposition 2.1. Let $\psi: \mathcal{P}(E) \rightarrow \mathcal{P}(E)$ be a translation invariant operator. There is a family of pairs of structuring elements $\left\{\left(A_{i}, B_{i}\right) \mid i \in I\right\}$ such that

$$
\psi(X)=\bigcup_{i \in I} X \circledast\left(A_{i}, B_{i}\right) .
$$




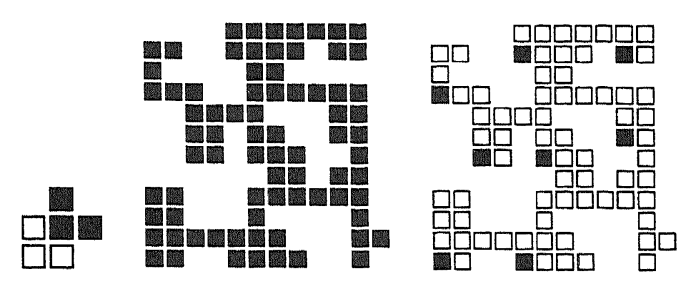

FIG. 1. Hit-or-miss operator for a discrete image. The structuring element (left; A comprises the black pixels, $B$ the white pixels) is chosen in such a way that the operator detects the lower-left corner points. The black pixels in the right image comprise the transformed image $X(A, B)$ of the original image $X$ (middle).

In fact, it is possible to give a characterization of the structuring elements required in this representation; see [1] and [17, §4.2].

2.2. Dilation and erosion. If we take $B=\varnothing$ in (2.1) we obtain the Minkowski difference

$$
X \ominus A=\left\{h \in E \mid A_{h} \subseteq X\right\},
$$

hereafter called the erosion of $X$ by $A$. Instead of $X \ominus A$ we also write $\varepsilon_{A}(X)$. Note that

$$
X \otimes(A, B)=(X \ominus A) \cap\left(X^{c} \ominus B\right) .
$$

Erosion is a translation invariant operator which is increasing, i.e.,

$$
X \subseteq Y \Rightarrow X \ominus A \subseteq Y \ominus A .
$$

Instead of (2.3) we can also write

$$
X \ominus A=\bigcap_{a \in A} X_{-a} .
$$

Another important operator is Minkowski addition given by

$$
X \oplus A=\bigcup_{a \in A} X_{a}
$$

also called the dilation of $X$ by $A$, and denoted by $\delta_{A}(X)$. Note that $X \oplus A=\{x+a \mid x \in$ $X, a \in A\}$. Dilation and erosion are illustrated in Fig. 2.

Defining the reflection of $A$ with respect to the origin by

$$
\check{A}=\{-a \mid a \in A\},
$$

we can also write

$$
X \oplus A=\left\{h \in E \mid \check{A}_{h} \cap X \neq \varnothing\right\} .
$$

It is clear that dilation defines an increasing translation invariant operator.

After these definitions we could give a long list of properties of erosion and dilation. However, we refrain from doing so and mention only those properties which we consider essential here. If $X_{i} \subseteq E$ for $i$ in some index set $I$, then

$$
\left(\bigcup_{i \in I} X_{i}\right) \oplus A=\bigcup_{i \in I}\left(X_{i} \oplus A\right),
$$

$$
\left(\bigcap_{i \in I} X_{i}\right) \ominus A=\bigcap_{i \in I}\left(X_{i} \ominus A\right),
$$



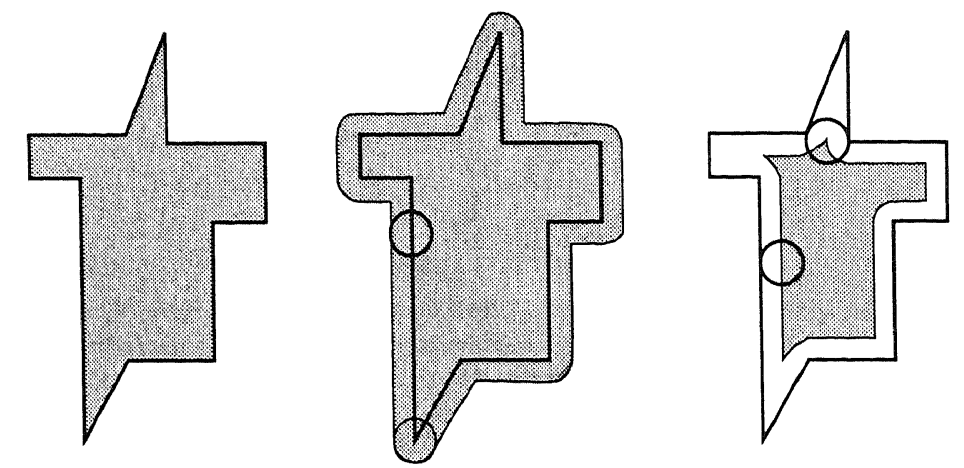

FIG. 2. From left to right (in grey): the original set $X$ and its dilation and erosion by a disk.

In the next section, where we discuss morphological operators in the context of complete lattices, we encounter these properties again.

If $X, A, B \subseteq E$ then

$$
\begin{aligned}
& (X \oplus A) \oplus B=X \oplus(A \oplus B), \\
& (X \ominus A) \ominus B=X \ominus(A \oplus B) .
\end{aligned}
$$

These properties show how to decompose erosions and dilations with large structuring elements in terms of erosions and dilations with smaller structuring elements. It is evident that "clever" decomposition procedures lay the basis for fast implementations of dilations and erosions. One has, for example,

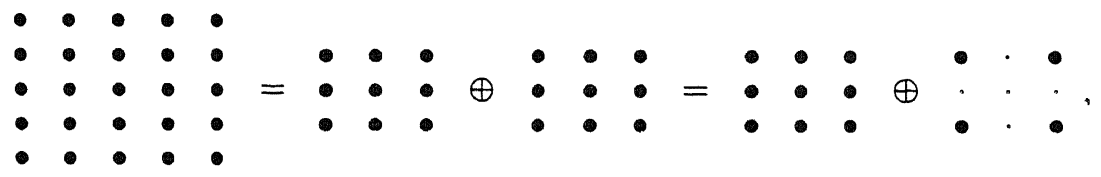

where the $\bullet$ 's represent the points in the structuring element. Note that in the ultimate decomposition one of the $3 \times 3$ squares is replaced by its extreme points. This reduces the number of operations in expressions like (2.9) and (2.10). For larger structuring elements this reduction becomes even more significant. Van den Boomgaard [46] uses this property to obtain logarithmic decompositions.

There is a considerable amount of literature about decomposition of structuring elements using Minkowski addition [49], [50]. We do not give any details here but only point out that discrete convexity plays an important role in these theories. To understand this we make the following observation: a compact set $A \subseteq \mathbb{R}^{d}$ is convex if and only if

$$
(r+s) A=r A \oplus s A,
$$

for $r, s>0$. The relation (2.11) also holds in the two-dimensional discrete case. In higher dimensions it is no longer true. We will meet property (2.11) again in $\S 5$ when we discuss granulometries.

We can specialize Proposition 2.1 to increasing translation invariant operators. To be specific, we define the kernel $\mathcal{V}(\psi)$ of a translation invariant operator $\psi$ by

$$
\mathcal{V}(\psi)=\{A \subseteq E \mid 0 \in \psi(A)\} .
$$

The following result is due to Matheron [28]. 
THEOREM 2.2. Let $\psi$ be an increasing translation invariant operator on $\mathcal{P}(E)$. Then

$$
\psi(X)=\bigcup_{A \in \mathcal{V}(\psi)} X \ominus A=\bigcap_{A \in \mathcal{V}\left(\psi^{*}\right)} X \oplus \check{A} .
$$

Here $\psi^{*}$ denotes the negative operator given by

$$
\psi^{*}(X)=\left[\psi\left(X^{c}\right)\right]^{c} .
$$

The kernel of an operator is too large to be of any practical use. Namely, since $\psi$ is increasing, $A \in \mathcal{V}(\psi)$ implies that also $A^{\prime} \in \mathcal{V}(\psi)$ for every set $A^{\prime} \supseteq A$. This observation motivated Maragos [26] to introduce the notion of a minimal kernel element and to look for (continuity) conditions on $\psi$ which guarantee that $\psi$ can be represented as a union of erosions by such minimal kernel elements.

Dilation and erosion are also negative operators in the following sense:

$$
(X \oplus A)^{c}=X^{c} \ominus \check{A} .
$$

In image processing terms, this means that dilation of the image foreground has the same effect as erosion of the background (with the reflected structuring element). But dilation and erosion are dual in yet another sense, namely

$$
Y \oplus A \subseteq X \Longleftrightarrow Y \subseteq X \ominus A
$$

for every pair of sets $X, Y \subseteq E$. This so-called adjunction relation forms the basis of the extension of morphological operators to complete lattices discussed in $\S 3$.

2.3. Openings and closings. Since the left-hand side of (2.14) is satisfied if we take $X=Y \oplus A$ we derive by substitution of this expression at the right-hand side:

$$
X \subseteq(X \oplus A) \ominus A=: X \bullet A
$$

In a similar way one can show that

$$
X \supseteq(X \ominus A) \oplus A=: X \circ A .
$$

We call $X \bullet A$ and $X \circ A$, respectively, the closing and opening of $X$ by $A$. From (2.15) and (2.16) we derive that

$$
X \oplus A \subseteq((X \oplus A) \ominus A) \oplus A=(X \oplus A) \circ A \subseteq X \oplus A,
$$

meaning that

$$
((X \oplus A) \ominus A) \oplus A=X \oplus A .
$$

Similar arguments give

$$
((X \ominus A) \oplus A) \ominus A=X \ominus A .
$$

As a consequence we get the following identities:

$$
(X \circ A) \circ A=X \circ A, \quad(X \bullet A) \bullet A=X \bullet A,
$$

saying that opening and closing are idempotent operators. We can derive the following alternative characterization of the opening:

$$
X \circ A=\bigcup\left\{A_{h} \mid h \in E \text { and } A_{h} \subseteq X\right\},
$$


i.e., $X \circ A$ comprises all translates of the structuring element $A$ which are contained in $X$. For the closing $X \bullet A$ we derive

$$
X \bullet A=\left\{k \in E \mid k \in \check{A}_{h} \Rightarrow \check{A}_{h} \cap X \neq \varnothing\right\} .
$$

Figure 3 illustrates the opening and the closing.

Opening and closing are negative operators in the following sense:

$$
(X \circ A)^{c}=X^{c} \bullet \check{A} .
$$
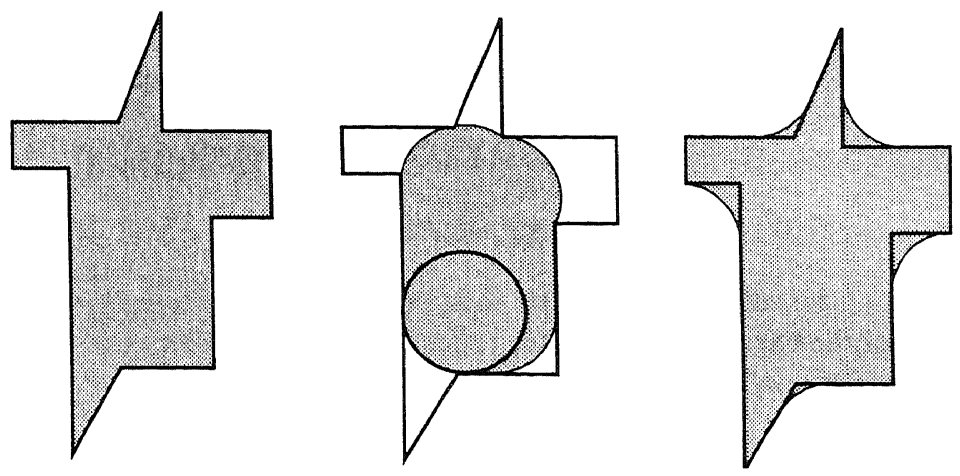

FIG. 3. A set and its opening and closing by a disk.

We now give the following general definition.

DEFINITION 2.3. An operator $\alpha: \mathcal{P}(E) \rightarrow \mathcal{P}(E)$ is called an opening if $\alpha$ is increasing, $\alpha^{2}=\alpha$ (idempotence), and $\alpha(X) \subseteq X$ for $X \subseteq E$ (anti-extensivity). An operator $\beta: \mathcal{P}(E) \rightarrow \mathcal{P}(E)$ is called a closing if $\beta$ is increasing, $\beta^{2}=\beta$ (idempotence), and $X \subseteq \beta(X)$ for $X \subseteq E$ (extensivity).

To distinguish the particular opening given by (2.16) from the general notion above we call the first a structural opening to emphasize that it uses only one structuring element. There are many openings which are not of structural type. In fact, if $\alpha_{i}$ is an opening for every $i$ in some index set $I$, then the operator given by $\alpha(X)=\bigcup_{i \in I} \alpha_{i}(X)$ is also an opening. Analogously, an arbitrary intersection of closings defines again a closing. In the literature one can find several alternative ways to construct openings. Refer in particular to [36], [37], and [41]. In $\S 5$ we discuss an iterative procedure to construct openings and closings.

The theorem below shows that structural openings and closings constitute a basis for all openings and closings. Define the invariance domain $\operatorname{Inv}(\psi)$ of an operator $\psi$ as the collection of all fixpoints of $\psi$, i.e., $\operatorname{Inv}(\psi)=\{X \subseteq E \mid \psi(X)=X\}$. It is easy to prove that the invariance domain of an opening is closed under unions. Moreover, if the opening is translation invariant then its invariance domain is closed under translations as well. The following result is due to Matheron [28].

THEOREM 2.4. (a) Let $\alpha$ be a translation invariant opening on $\mathcal{P}(E)$; then

$$
\alpha(X)=\bigcup_{A \in \operatorname{Inv}(\alpha)} X \circ A
$$

for every $X \subseteq E$.

(b) Let $\beta$ be a translation invariant closing on $\mathcal{P}(E)$; then

$$
\beta(X)=\bigcap_{A \in \operatorname{Inv}(\beta)} X \bullet A
$$

for every $X \subseteq E$. 
Openings and closings play a rather prominent role in mathematical morphology. We shall see one particular application in $\S 5$, where we discuss granulometries and size distributions. A second application is the cleaning of images. If an image is corrupted by noise, one can try to recover it by applying openings and/or closings. This is the key idea underlying the so-called alternating sequential filters. We point out here that, unlike in classical signal processing, in mathematical morphology the name "filter" is preserved for those operators which are increasing and idempotent. Consider a family of structuring elements $A_{n}, n \geq 1$, such that $A_{n}$ is $A_{n-1}$-open, i.e., $A_{n} \circ A_{n-1}=A_{n}$. Let $\alpha_{n}$ and $\beta_{n}$ be the opening and closing by $A_{n}$, respectively. Then

$$
\alpha_{n} \alpha_{n-1}=\alpha_{n} \text { and } \beta_{n} \beta_{n-1}=\beta_{n} .
$$

We define the alternating sequential filter of order $n$ by

$$
v_{n}=\left(\beta_{n} \alpha_{n}\right)\left(\beta_{n-1} \alpha_{n-1}\right) \cdots\left(\beta_{1} \alpha_{1}\right) .
$$

It has been shown [41] that $v_{n}$ is idempotent, whence the name filter. Furthermore, the filters $\nu_{n}$ satisfy the semigroup property

$$
\nu_{n} v_{m} \leq \nu_{m}=v_{m} v_{n} \quad \text { if } m \geq n .
$$

Here $\phi \leq \psi$ means that $\phi(X) \subseteq \psi(X)$ for $X \subseteq E$. By duality, one also obtains a filter if one interchanges the $\alpha$ 's and the $\beta$ 's in the expression for $\nu_{n}$. In Fig. 4 we present an example which illustrates clearly the noise-cleaning effect of alternating sequential filters. Here $A_{n}$ is the $(2 n+1) \times(2 n+1)$-square in which case the condition " $A_{n}$ is $A_{n-1}$-open" is obviously satisfied.

The following remark is in order here: so far we have only discussed operators for binary images. Yet, in Fig. 4 we apply some of these operators to grey-scale images. In $\S 6$ it will be explained how any set operator can be applied to a grey-scale image by means of a thresholding procedure.

2.4. Morphological operators and Boolean functions. We conclude this section with a brief discussion of the relation between morphological operators and Boolean functions. A thorough exposition can be found in [17]; see also [45]. For a comprehensive account of Boolean functions the reader should refer to Harrison [11]. We restrict ourselves to the case $E=\mathbb{Z}^{d}$.

If, for $d=2$, one wants to perform a dilation with the $3 \times 3$ square

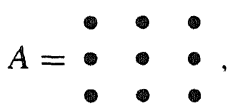

one has to carry out the following procedure for every pixel $h$ : look at the values of $X(\cdot)$ (the characteristic function of $X$ ) at $h$ and its eight neighbors; if at least one of these values is 1 then $h$ lies in the dilated set. One can easily generalize this to arbitrary Boolean functions. Assume that $A$ is a structuring element containing $n$ points $a_{1}, a_{2}, \ldots, a_{n}$, and that $b$ is a Boolean function of $n$ variables. Define the operator $\psi_{b}$ by

$$
\psi_{b}(X)=\left\{h \in \mathbb{Z}^{d} \mid b\left(X\left(a_{1}+h\right), X\left(a_{2}+h\right), \ldots, X\left(a_{n}+h\right)\right)=1\right\} .
$$

Note that $\psi_{b}$ also depends on $A$. We say that the translation invariant operator $\psi: \mathcal{P}\left(\mathbb{Z}^{d}\right) \rightarrow \mathcal{P}\left(\mathbb{Z}^{d}\right)$ is a finite window operator if there exists a finite set $A \subseteq \mathbb{Z}^{d}$ such that

$$
h \in \psi(X) \Longleftrightarrow h \in \psi\left(X \cap A_{h}^{\prime}\right)
$$




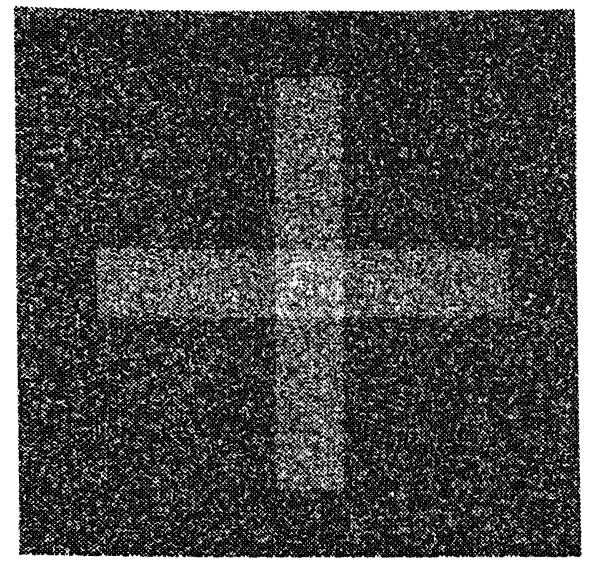

(a)

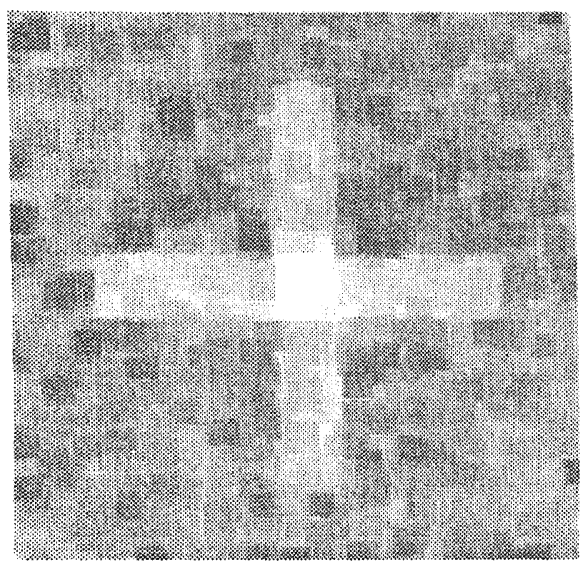

(c)

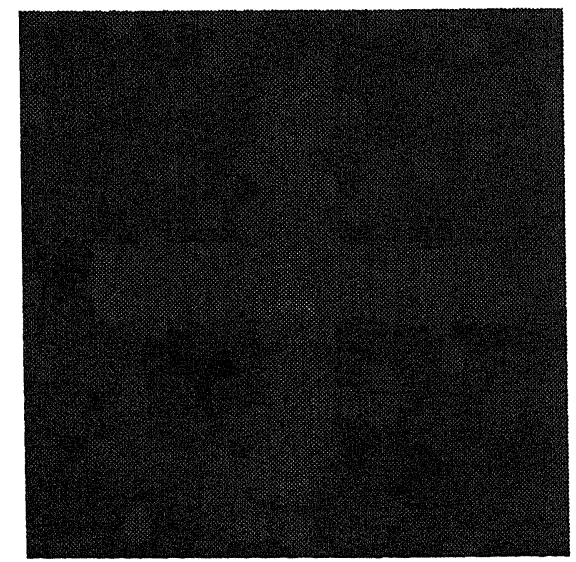

(b)

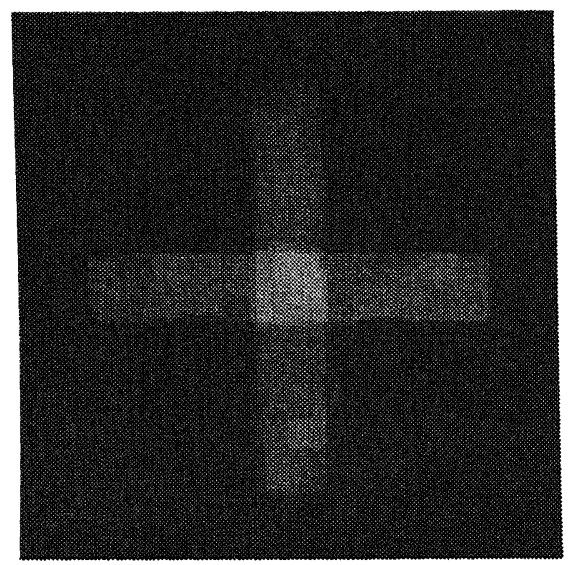

(d)

FIG. 4. Let $A_{n}$ be the $(2 n+1) \times(2 n+1)$ square. (a) Original image $X ;$ (b) opening $\alpha_{5}(X)=X \circ A_{5}$; (c) closing $\beta_{5}(X)=X \bullet A_{5}$; (d) alternating sequential filters $v_{5}(X)$.

for every $h \in \mathbb{Z}^{d}, X \subseteq \mathbb{Z}^{d}$, and $A^{\prime} \supseteq A$. It is not difficult to show that every translation invariant finite window operator is of the form (2.23); see, e.g., [17]. The dilation can be represented by means of a Boolean function of the form $b\left(x_{1}, \ldots, x_{n}\right)=x_{1}+\cdots+x_{n}$, where "+" denotes the logic "OR." If $b$ is a positive (=increasing) Boolean function, then $\psi_{b}$ is increasing. If $b$ is additive (like in the example above), respectively, multiplicative, then $\psi_{b}$ $s$ a dilation, respectively, an erosion.

As a first example consider the hit-or-miss operator $X \rightarrow X \otimes(B, C)$ where $B \cap C=$ x. This operator can be represented in terms of a Boolean function as follows. Let $B=$ $\left\{a_{1}, a_{2}, \ldots, a_{m}\right\}$ and $C=\left\{a_{m+1}, a_{m+2}, \ldots, a_{n}\right\}$; define $A=B \cup C$ and

$$
b\left(x_{1}, \ldots, x_{n}\right)=x_{1} \cdot x_{2} \ldots \ldots x_{m} \cdot \overline{x_{m+1}} \cdot \overline{x_{m+2}} \cdots \overline{x_{n}} .
$$

Here $x \cdot y$ stands for " $x$ AND $y$," and $\bar{x}$ means "NOT $x$." Using (2.23) we find that $h \in \psi_{b}(X)$ if and only if $a_{1}+h, a_{2}+h, \ldots, a_{m}+h \in X$, and $a_{m+1}+h, a_{m+2}+h, \ldots, a_{n}+h \notin X$, that is, $B_{h} \subseteq X$ and $C_{h} \subseteq X^{c}$. This shows that $\psi_{b}(X)=X(B, C)$. 
As a second example we discuss rank operators, sometimes called order statistics. Let $r_{k}, k \leq n$ be the positive Boolean function of $n$ variables which takes the value 1 if at least $k$ variables are equal to 1 and 0 otherwise. It is clear that $r_{1}\left(x_{1}, \ldots, x_{n}\right)=x_{1}+\cdots+x_{n}$ and $r_{n}\left(x_{1}, \ldots, x_{n}\right)=x_{1} \cdots x_{n}$. If $A$ is a structuring element with $n$ points and if $k \leq n$ then we define the rank operator $\rho_{A, k}$ as the operator given by (2.23) with $b=r_{k}$. It is evident that $\rho_{A, 1}(X)=X \oplus \check{A}$ and $\rho_{A, n}(X)=X \ominus A$. Furthermore,

$$
\rho_{A, n} \leq \rho_{A, n-1} \leq \cdots \leq \rho_{A, 1} .
$$

If $n$ is odd and $k=(n+1) / 2$ then $\rho_{A, k}$ is usually referred to as the median operator. It is easy to check that the median operator is self-dual. (An operator $\psi$ is called self-dual if $\psi^{*}=\psi$.) In Fig. 5 we have illustrated the rank operator for different values of $k$.

\section{An algebraic approach.}

3.1. Motivation. From the previous section, mathematical morphology appears as a collection of image transforms based on set-theoretical operations such as union, intersection, and complementation. Furthermore, these transforms are built in such a way that they are translation invariant. Although this paper deals exclusively with transforms mapping images onto images, the ultimate goal of mathematical morphology (and, in fact, of most image analysis systems) in practice is to arrive at a recognition or classification of an image or its constituting parts. For that purpose one has to perform certain measurements, i.e., transforms mapping an image onto a number or a collection of numbers (e.g., the moments of a size distribution). The power of mathematical morphology is partially due to the fact that it has successfully integrated techniques from integral geometry, stereology, and stochastic geometry.

In the previous section only binary images have been discussed. In this case the image space can be modeled appropriately by $\mathcal{P}(E), E$ being the Euclidean space $\mathbb{R}^{d}$ or the discrete space $\mathbb{Z}^{d}$. But depending on the kind of images one wants to consider, the sort of information one needs to extract, or the mathematical techniques one requires for further analysis, other object spaces may be more appropriate. For instance, integral geometry relies heavily upon the theory of convex sets [9], [28], meaning that if one is about to use integral geometrical tools, a restriction to convex sets may be necessary, or at least helpful. In stochastic geometry one has to supply the underlying space with a topology. This is possible if one takes the space of closed sets or the space of compact sets as the image space [28]. Furthermore, it is important to extend mathematical morphology to grey-scale images which can be modelled mathematically as functions. Here several possibilities emerge: we can choose continuous, discrete or finite grey-value sets, we can restrict ourselves to (semi-) continuous functions, convex functions, or functions with compact domains.

Besides this large variation in object spaces there is yet another generalization which is quite important. In fact, it is by no means obvious why morphological operators have to be translation invariant. One can think of various situations (e.g., radar imaging) where rotation invariance is more appropriate. Furthermore, in certain problems perspective transformations come in naturally; think, for instance, of the problem of monitoring the traffic on a highway with a camera at a fixed position. It is obvious that in such a configuration the detection algorithms should take the distance between the camera and the object (e.g., a car) into account.

These considerations are sufficient motivation to think about an abstraction of mathematical morphology which includes all mentioned object spaces and allows the generalization of translation invariance to other transformation groups. Such an abstraction was initiated by Matheron and Serra in [41]. They argued that the structure of a complete lattice is the appropriate framework for a general theory of mathematical morphology. Their work, however, 

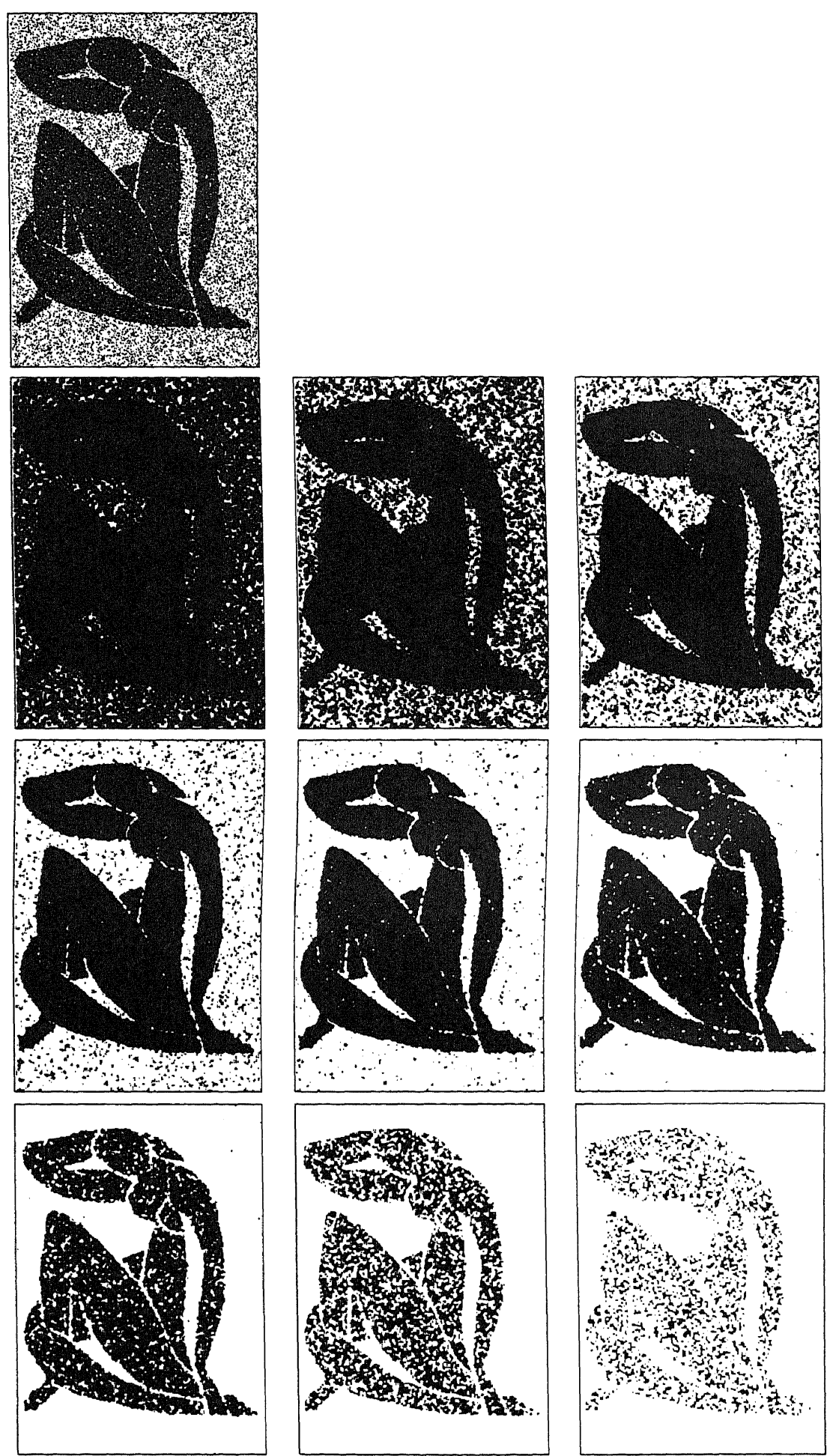

FIG. 5. Rank operators applied to a noisy image X (black pixels; H. Matisse's "Nu Bleu II," 1952). From left to right and top to bottom: the original image $X$ and its transforms $\rho_{A, n}(X)$, with $n=1,2, \ldots, 9$. Here $A$ is the $3 \times 3$ square consisting of 9 pixels. 
did not include invariance under transformation groups. These extensions were first made by Heijmans and Ronse [19], [37] for Abelian transformation groups. Later this was generalized to non-Abelian transformation groups by Roerdink [33], [34]. A comprehensive overview of the complete lattice framework of morphology can be found in our book [17]. In this section we give a short overview of the main results in [19] and [37] and illustrate them by a concrete example. In fact, $\S 6$, which discusses morphology for grey-scale functions, comprises another application.

3.2. Complete lattices. Although we expect that most readers are familiar with the basic theory of complete lattices, we briefly recall some basic notions. A complete lattice is a set $\mathcal{L}$ with a partial ordering " $\leq$ " such that every subset $\mathcal{H}$ of $\mathcal{L}$ has a least upper bound $\bigvee \mathcal{H}$, called supremum, and a greatest lower bound $\wedge \mathcal{H}$, called infimum. The least and greatest elements of $\mathcal{L}$ are denoted, respectively, by $O$ and $I$. Refer to Birkhoff [2] for a general account on the theory of complete lattices. From now on we denote by $\mathcal{L}$ an arbitrary complete lattice.

A trivial but very useful fact is the observation that $\mathcal{L}$, endowed with the opposite ordering $X \leq^{\prime} Y$ iff $Y \leq X$, is also a complete lattice, called the opposite or dual of $\mathcal{L}$, and denoted by $\mathcal{L}^{\prime}$. To every definition, statement, property, etc. referring to $\mathcal{L}$ there corresponds a dual one referring to $\mathcal{L}^{\prime}$, interchanging the role of $\leq$ and $\leq^{\prime}$. This principle is known as the duality principle.

We mention some examples of complete lattices which are relevant in the context of mathematical morphology. We have introduced the power set $\mathcal{P}(E)$ in the previous section; this is a complete lattice if we take set inclusion as the partial ordering. If $E$ is a topological space, then the closed sets $\mathcal{F}(E)$ ordered by inclusion constitute a complete lattice; in this case the supremum of a collection $X_{i}, i \in I$, of closed sets is $\overline{\bigcup_{i \in I} X_{i}}$, where the bar denotes closure. Analogously, the open sets constitute a complete lattice (note that this lattice is isomorphic to the opposite of $\mathcal{F}(E)$ ). If $E$ is a real vector space, then $\mathcal{C}(E)$, the convex subsets of $E$ ordered by inclusion, define a complete lattice. The infimum is the ordinary set intersection and the supremum is given by

$$
\bigvee_{i \in I} X_{i}=\operatorname{co}\left(\bigcup_{i \in I} X_{i}\right) .
$$

Here $\operatorname{co}(\cdot)$ denotes the convex hull. If $E$ is a nonvoid space and $\mathcal{T}$ a complete lattice then we denote by $\operatorname{Fun}(E, \mathcal{T})$ the space of all functions mapping $E$ into $\mathcal{T}$. With the pointwise ordering of $\mathcal{T}\left(F \leq F^{\prime}\right.$ iff $F(x) \leq F^{\prime}(x)$ for every $x \in E$ ) this space becomes a complete lattice. This lattice plays an important role in $\S 6$. A choice for $\mathcal{T}$ which is particularly important is $\mathcal{T}=\overline{\mathbb{R}}:=\mathbb{R} \cup\{-\infty,+\infty\}$.

A lattice $\mathcal{L}$ is called distributive if

$$
\begin{aligned}
& X \wedge(Y \vee Z)=(X \wedge Y) \vee(X \wedge Z), \\
& X \vee(Y \wedge Z)=(X \vee Y) \wedge(X \vee Z),
\end{aligned}
$$

for all $X, Y, Z \in \mathcal{L}$. It is called modular if the identity

$$
X \vee(Y \wedge Z)=(X \vee Y) \wedge Z \text { if } X \leq Z
$$

is satisfied for all $X, Y, Z \in \mathcal{L}$. Every distributive lattice is modular, but the converse is in general not true.

If $X, Y \in \mathcal{L}$ are such that $X \wedge Y=0$ and $X \vee Y=I$, then $Y$ is called the complemen of $X$. We write $Y=X^{c}$. A Boolean lattice is a complete distributive lattice in which ever. element has a complement. 
3.3. Operators on complete lattices. An operator $\psi: \mathcal{L} \rightarrow \mathcal{L}$ is increasing if it preserves the ordering structure, that is, $X \leq Y$ implies that $\psi(X) \leq \psi(Y)$. It is called decreasing if it reverses the ordering structure. The increasing operators on $\mathcal{L}$ define a complete lattice under the ordering

$$
\psi \leq \psi^{\prime} \Longleftrightarrow \psi(X) \leq \psi^{\prime}(X) \quad \text { for all } X \in \mathcal{L} .
$$

A mapping $\psi$ on $\mathcal{L}$ is called a lattice automorphism if $\psi$ is an increasing bijection. Then $\psi\left(\bigvee_{i \in I} X_{i}\right)=\bigvee_{i \in I} \psi\left(X_{i}\right)$ and $\psi\left(\bigwedge_{i \in I} X_{i}\right)=\bigwedge_{i \in I} \psi\left(X_{i}\right)$, for every collection $X_{i}$. A decreasing bijection is called a dual automorphism. A dual automorphism which satisfies

$$
\psi^{2}=i d
$$

id denoting the identity operator, is called a negation. If $\psi$ is a negation then $X^{*}=\psi(X)$ is called the negative of $X$; although such nomenclature is in general ambiguous due to the fact that negations are not necessarily unique, it will not give rise to any confusion. Note that on a Boolean lattice the complement operator is a negation. On Fun $(E, \overline{\mathbb{R}})$ the mapping $F \rightarrow-F$ defines a negation. Note, however, that $-F$ is not the complement of $F$. In fact, it is not difficult to verify that the only functions which have a complement are those functions which attain only the values $-\infty$ and $+\infty$. This means, in particular, that $\operatorname{Fun}(E, \overline{\mathbb{R}})$ is not a Boolean lattice.

If $\psi$ is an operator mapping $\mathcal{L}$ into another complete lattice $\mathcal{M}$, and if both lattices have a negation, then we define the negative operator $\psi^{*}: \mathcal{L} \rightarrow \mathcal{M}$ by

$$
\psi^{*}(X)=\left[\psi\left(X^{*}\right)\right]^{*}
$$

Note that $\psi^{*}$ is increasing (decreasing) if and only if $\psi$ is increasing (decreasing).

3.4. Adjunctions. The basis for the definition of morphological operators on complete lattices is formed by the concept of an adjunction.

DEFINITION 3.1. Let $\mathcal{L}, \mathcal{M}$ be complete lattices, let $\varepsilon$ be an operator from $\mathcal{L}$ into $\mathcal{M}$, and $\delta$ an operator from $\mathcal{M}$ into $\mathcal{L}$. The pair $(\varepsilon, \delta)$ is called an adjunction between $\mathcal{L}$ and $\mathcal{M}$ if

$$
\delta(Y) \leq X \Longleftrightarrow Y \leq \varepsilon(X),
$$

for every $X \in \mathcal{L}$ and $Y \in \mathcal{M}$. In this case $\delta$ is called $a$ dilation and $\varepsilon$ an erosion.

We summarize some basic properties. For a systematic treatment of adjunctions we refer to [19] and [17].

PROPOSITION 3.2. Let $(\varepsilon, \delta)$ be an adjunction between $\mathcal{L}$ and $\mathcal{M}$. Then

(a) $\varepsilon(I)=I$ and $\delta(O)=O$.

(b) $\varepsilon\left(\bigwedge_{i \in I} X_{i}\right)=\bigwedge_{i \in I} \varepsilon\left(X_{i}\right)$ for every collection $X_{i} \in \mathcal{L}(i \in I)$.

(c) $\delta\left(\bigvee_{i \in I} Y_{i}\right)=\bigvee_{i \in I} \delta\left(Y_{i}\right)$ for every collection $Y_{i} \in \mathcal{M}(i \in I)$.

(d) $\varepsilon \delta \geq i \mathrm{~d}_{\mathcal{M}}$.

(e) $\delta_{\varepsilon} \leq i d_{\mathcal{L}}$.

(f) $\varepsilon \delta \varepsilon=\varepsilon$.

(g) $\delta \varepsilon \delta=\delta$.

(h) $\varepsilon(X)=\bigvee\{Y \in \mathcal{M} \mid \delta(Y) \leq X\}$.

(i) $\delta(Y)=\bigwedge\{X \in \mathcal{L} \mid Y \leq \varepsilon(X)\}$.

For (2.14) it follows that the pair $\left(\varepsilon_{A}, \delta_{A}\right)$ defines an adjunction on $\mathcal{P}(E)$.

PROPOSITION 3.3. With every erosion $\varepsilon: \mathcal{L} \rightarrow \mathcal{M}$ one can associate a unique dilation $\delta: \mathcal{M} \rightarrow \mathcal{L}$ such that $(\varepsilon, \delta)$ forms an adjunction between $\mathcal{L}$ and $\mathcal{M}$. Dually, with every 
dilation $\delta: \mathcal{M} \rightarrow \mathcal{L}$ one can associate a unique erosion $\varepsilon: \mathcal{L} \rightarrow \mathcal{M}$ such that $(\varepsilon, \delta)$ forms an adjunction between $\mathcal{L}$ and $\mathcal{M}$.

PROPOSITION 3.4. If $\left(\varepsilon_{i}, \delta_{i}\right)$ is an adjunction for every $i \in I$, then $\left(\bigwedge_{i \in I} \varepsilon_{i}, \bigvee_{i \in I} \delta_{i}\right)$ is an adjunction as well.

PROPOSITION 3.5. Let $\mathcal{L}, \mathcal{M}$ be complete lattices with a negation and let $(\varepsilon, \delta)$ be an adjunction between $\mathcal{L}$ and $\mathcal{M}$. Then $\left(\delta^{*}, \varepsilon^{*}\right)$ defines an adjunction between $\mathcal{M}$ and $\mathcal{L}$.

3.5. T-invariance. Let $\mathbb{T}$ be an Abelian group of automorphisms on $\mathcal{L}$; an operator $\psi$ on $\mathcal{L}$ is called $\mathbb{T}$-invariant if

$$
\psi \tau=\tau \psi, \quad \tau \in \mathbb{T}
$$

A $\mathbb{T}$-invariant operator is called a $\mathbb{T}$-operator. Similarly, a $\mathbb{T}$-invariant dilation is called a $\mathbb{T}$-dilation. If $\mathcal{L}=\mathcal{P}\left(\mathbb{R}^{d}\right)$ and $\mathbb{T}$ is the group of translations, then "T-invariant" means "translation invariant." If $(\varepsilon, \delta)$ is an adjunction on $\mathcal{L}$ and if $\varepsilon$ or $\delta$ is $\mathbb{T}$-invariant, then both operators are $\mathbb{T}$-invariant. If $\tau \in \mathbb{T}$, then $\left(\tau^{-1}, \tau\right)$ is a $\mathbb{T}$-adjunction. Together with Proposition 3.4 this means that $\left(\bigwedge_{i \in I} \tau_{i}^{-1}, \bigvee_{i \in I} \tau_{i}\right)$ is a $\mathbb{T}$-adjunction if $\tau_{i} \in \mathbb{T}$ for $i \in I$. Below we give assumptions under which every $\mathbb{T}$-adjunction is of this form. This Basic Assumption holds in particular for the translation invariant case discussed in the previous section. Essentially, two properties of the space $\mathcal{P}(E)$ are used here: (1) every set is a union of points, and (2) the translation group acts simply transitively on the points. The latter means that for every two points there is a unique translation mapping the first point onto the second. Essentially, the Basic Assumption below generalizes these two properties.

A subset $\ell$ of $\mathcal{L}$ is called a sup-generating family if every element of $\mathcal{L}$ can be obtained as a supremum of elements of $\ell$. As a special example we mention the set of atoms in an atomic lattice; see [2]. Note that $\mathcal{P}(E)$ is atomic, the atoms being the singletons.

BASIC ASSUMPTION 3.6. $\mathcal{L}$ is a complete lattice which possesses a sup-generating family $\ell$, and $\mathbb{T}$ is an Abelian automorphism group on $\mathcal{L}$ such that (i) $\ell$ is invariant under $\mathbb{T}$, (ii) $\mathbb{T}$ is simply transitive on $\ell$.

From now on we assume that the Basic Assumption holds. We indicate how to define Minkowski addition and subtraction on $\mathcal{L}$. First we fix an origin $o \in \mathcal{L}$. For every $h \in \ell$ there is a unique $\tau_{h} \in \mathbb{T}$ which sends $o$ to $h$. Define Minkowski addition and subtraction as follows:

$$
\begin{aligned}
& \delta_{A}(X):=X \oplus A=\bigvee_{a \in \ell(A)} \tau_{a}(X), \\
& \varepsilon_{A}(X):=X \ominus A=\bigwedge_{a \in \ell(A)} \tau_{a}^{-1}(X),
\end{aligned}
$$

for $X, A \in \mathcal{L}$. Here $\ell(A)=\{a \in \ell \mid a \leq A\}$. Note that $\delta_{A}=\bigvee_{a \in \ell(A)} \tau_{a}$ and that $\varepsilon_{A}=$ $\bigwedge_{a \in \ell(A)} \tau_{a}^{-1}$.

PROPOSITION 3.7. For every $A \in \mathcal{L}$, the pair $\left(\varepsilon_{A}, \delta_{A}\right)$ forms a $\mathbb{T}$-adjunction on $\mathcal{L}$. Moreover, any $\mathbb{T}$-adjunction has this form.

It is rather straightforward to establish properties similar to those stated in the previous section, including the representation Theorem 2.2. We will not do so, however; the interested reader can find additional results in [19] or [17].

Taking Definition 2.3 as a starting point, the concept of the opening and closing can easily be extended to the given abstract framework. As in the translation invariant case the concept of a structural opening plays an important role. A comprehensive discussion can be found in [37] and [17].

We conclude this section, mainly intended as a trend-setter, with the following example. Take $E=\mathbb{R}^{2} \backslash\{0\}$ and $\mathcal{L}=\mathcal{P}(E)$. Furthermore, let $\mathbb{T}$ be the Abelian group of rotations and 
scalar multiplications with respect to the origin. It is obvious that the Basic Assumption is satisfied, so we can define Minkowski addition and subtraction in this case. Note that here the size of a translate of the structuring element depends on its distance from the origin; see Fig. 6.
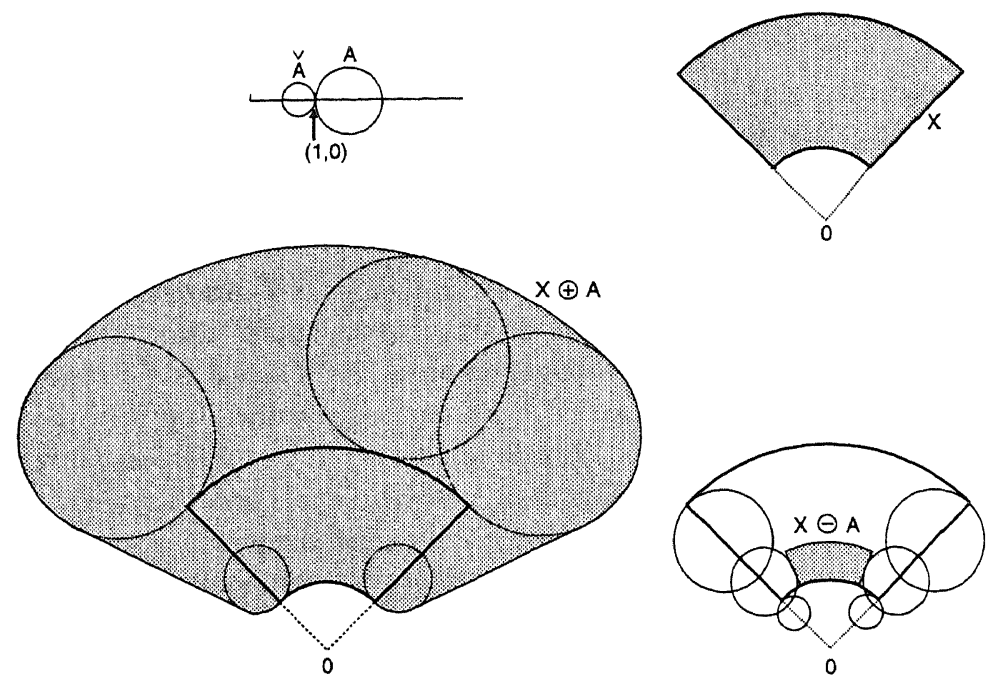

FIG. 6. The effect of a dilation and erosion which are invariant under rotation and scalar multiplication. To compute the erosion we have used $X \Theta A=\left(X^{c} \oplus \check{A}\right)^{c}$.

If the assumption that $\mathbb{T}$ is Abelian is dropped then we have to distinguish between two different cases, (i) the simply transitive case, and (ii) the multiply transitive case. Needless to say, the second case is considerably more complex than the first; refer to [33], [34], and [17] for a detailed discussion. As an important application of the non-Abelian, multiply transitive case we mention the rotation-translation group on $\mathcal{P}\left(\mathbb{R}^{2}\right)$.

\section{Morphological filtering.}

4.1. Motivation. In $\S 2$ we have already indicated the importance of openings and closings (and alternating sequential filters) with respect to image filtering, i.e., the preprocessing of an image with the intention to remove noise, sharpen edges, enhance contrasts, etc. Suppose we apply operator $\psi$ to image $X$ in order to make it more suited for subsequent analysis. If $\psi$ is not idempotent then $\psi^{2}(X) \neq \psi(X)$ in general. If $\psi(X)$ contains less noise than $X$, then it seems reasonable to assume that $\psi^{2}(X)$ contains less noise than $\psi(X)$. This suggests that $\psi$ should be applied until the result remains unchanged. Denoting by $\tilde{\psi}(X)$ the final result of such an iteration procedure (presuming that it converges eventually) it is clear that $\psi \tilde{\psi}=\tilde{\psi}$, and hence that $\tilde{\psi}$ is idempotent. Obviously, idempotence is a desirable property in any image filtering procedure. As we have seen, openings, closings, and alternating sequential filters satisfy this requirement, which makes them suited for the removal of noise.

The iteration procedure above raises the following two questions: (1) What kind of convergence has to be considered? (2) For which operators $\psi$ does iteration lead to an idempotent operator?

Before we deal with such problems we present some basic concepts from the theory of morphological filtering. Again, we do not pursue completeness, but rather we intend to give the reader a flavor of the mathematical concepts and tools which play a role. Throughout this section we assume that our image space $\mathcal{L}$ is an arbitrary complete lattice. Unlike in the 
previous section we do not assume our operators to be invariant under some transformation group.

4.2. Basic concepts. The theory of morphological filtering was initiated by Matheron. His main ideas are contained in Chapter 6 of Serra [41]; see also other chapters in this book. Most of the concepts introduced below can be found in this reference; see also [13], [17], [20], [25], and [37]. The tutorial paper by Serra and Vincent [42] comprises a nice introduction to the theory of morphological filters. In $\S 2$ we have seen that an arbitrary union of openings is again an opening. This is not true for intersections; furthermore, the composition of two openings is in general not an opening. This shows in particular that the property of idempotence is not preserved under suprema, infima, and compositions: if $\phi, \psi$ are increasing idempotent operators, then none of the operators $\phi \vee \psi, \phi \wedge \psi, \phi \psi$ is in general idempotent. Though this is rather unfortunate, it does not mean that nothing can be said. In fact, taking suprema one part of the idempotence property is preserved. To be specific, $(\phi \vee \psi)^{2} \geq \phi^{2} \vee \psi^{2}=\phi \vee \psi$; we say that $\phi \vee \psi$ is an overfilter.

DEFINITION 4.1. Let $\psi$ be an increasing operator on $\mathcal{L}$. We say that $\psi$ is

(a) $a$ (morphological) filter if $\psi^{2}=\psi$;

(b) an underfilter if $\psi^{2} \leq \psi$;

(c) an overfilter if $\psi^{2} \geq \psi$;

(d) an inf-overfilter if $\psi=\psi($ id $\wedge \psi)$;

(e) a sup-underfilter if $\psi=\psi(i d \vee \psi)$;

(f) a strong filter if $\psi$ is both an inf-overfilter and a sup-underfilter;

(g) an opening if $\psi$ is increasing, idempotent, and anti-extensive ( $\psi \leq \mathrm{id})$;

(h) a closing if $\psi$ is increasing, idempotent, and extensive ( $\psi \geq \mathrm{id}$ ).

The latter two definitions generalize Definition 2.3 to the complete lattice framework. From Proposition 3.2(d)-(g) it follows immediately that $\delta \varepsilon$ is an opening on $\mathcal{L}$ and that $\varepsilon \delta$ is a closing on $\mathcal{M}$ if $(\varepsilon, \delta)$ is an adjunction between $\mathcal{L}$ and $\mathcal{M}$.

It is clear that underfilters and overfilters are dual concepts in the sense of the duality principle. The same remark applies to inf-overfilters and sup-underfilters. For that reason we restrict ourselves to (inf-) overfilters. We start with some basic properties.

- every inf-overfilter is an overfilter;

- every extensive operator is an inf-overfilter;

- $\psi$ is a filter iff it is both an underfilter and an overfilter;

- the set of both overfilters and inf-overfilters is closed under suprema;

- the set of (inf-) overfilters is closed under self-composition (i.e., if $\psi$ is an (inf-) overfilter then $\psi^{n}$ is such as well for every integer $n \geq 1$ );

- openings and closings are strong filters;

- if $\psi$ is an inf-overfilter then id $\wedge \psi$ is an opening.

This last property is very important because it provides a powerful technique for the construction of openings: see [35] and [37] for more details.

We define $\breve{\psi}$ as the largest opening $\leq \psi$, that is, $\check{\psi}=\bigvee\{\alpha \mid \alpha$ is an opening and $\alpha \leq \psi\}$. Since every supremum of openings is again an opening this definition makes sense. It can be shown that

$$
\operatorname{Inv}(\check{\psi})=\operatorname{Inv}(i d \wedge \psi)=\{X \in \mathcal{L} \mid \psi(X) \geq X\}
$$

Let $\mathcal{C}$ be the smallest collection of increasing operators on $\mathcal{L}$ which contains id $\wedge \psi$ and which is closed under compositions and infima. It is easy to see that $\breve{\psi} \leq \phi \leq$ id $\wedge \psi$ for every $\phi \in \mathcal{C}$. Let $\alpha:=\inf \mathcal{C}$; then $\alpha \in \mathcal{C}$ because $\mathcal{C}$ is closed under infima. In particular, $\alpha^{2} \leq \alpha$ since $\alpha \leq i d$. On the other hand, $\alpha^{2} \in \mathcal{C}$, and by the very definition of $\alpha$ this means that 
$\alpha^{2} \geq \alpha$. Therefore $\alpha^{2}=\alpha$ and we conclude that $\alpha$ is an opening $\leq \psi$. Since $\breve{\psi}$ is the largest such opening and $\alpha \geq \check{\psi}$ we may conclude that $\alpha=\breve{\psi}=\inf \mathcal{C}$.

For the mathematical connoisseurs we point out that the mapping $\psi \rightarrow \breve{\psi}$ defines an opening on the complete lattice of increasing operators on $\mathcal{L}$. Dually, we define $\hat{\psi}$ as the smallest closing $\geq \psi$. The proof of the next result can be found in [41, Chap. 6]; see also [17, Chap. 12].

PROPOSITION 4.2. Let $\psi$ be an increasing operator on $\mathcal{L}$.

(a) $\breve{\psi} \psi$ is an overfilter. It is the largest overfilter $\leq \psi$.

(b) If $\psi$ is an underfilter then $\breve{\psi} \psi$ is a filter. It is the largest filter $\leq \psi$.

(c) $\psi \dot{\psi}$ is an inf-overfilter. It is the largest inf-overfilter $\leq \psi$.

(d) Assume that $\mathcal{L}$ is modular. If $\psi$ is a sup-underfilter then $\psi \tilde{\psi}$ is a strong filter.

Note that (a) can be restated as follows: the mapping $\psi \rightarrow \breve{\psi} \psi$ on the complete lattice of increasing operators on $\mathcal{L}$ is an opening with invariance domain the set of overfilters. We mention the following important consequence of this result.

COROLLARY 4.3. The filters on $\mathcal{L}$ define a complete lattice.

Here we have provided the set of filters with the ordering given in (3.1). To prove this result, take an arbitrary collection $\psi_{i}$ of filters and define the underfilter $\lambda$ and the overfilter $\mu$, respectively, by

$$
\lambda:=\bigwedge_{i \in I} \psi_{i} \text { and } \mu:=\bigvee_{i \in I} \psi_{i}
$$

Then, by Proposition 4.2(b), $\check{\lambda} \lambda$ is a filter, the largest filter $\leq \psi_{i}$, for every $i \in I$, and therefore the infimum of the $\psi_{i}$ in the lattice of filters. Similarly, $\hat{\mu} \mu$ is the supremum of the $\psi_{i}$ in this lattice. We can use similar arguments to show that the set of strong filters, the set of openings, and the set of closings constitute complete lattices.

If $\mathcal{L}$ has a negation, then the mapping $\psi \rightarrow \psi^{*}$ given by (3.2) transforms an (inf-) overfilter into a (sup-) underfilter, an opening into a closing, and a (strong) filter into a (strong) filter. A filter $\psi$ is called self-dual if $\psi^{*}=\psi$. In image processing self-dual filters are rather popular since they treat fore- and background of an image identically. The example at the end of this section deals with the construction of a self-dual filter. A general construction method is described in [17, Chap. 13].

4.3. Iteration. The above considerations show the need for methods to compute the socalled lower and upper envelopes $\breve{\psi}$ and $\hat{\psi}$. Below we explain that under certain assumptions on $\psi$, the lower envelope can be computed through iteration of the anti-extensive operator id $\wedge \psi$. Dually, iteration of $i d \vee \psi$ yields the upper envelope $\hat{\psi}$.

Assume that $\psi$ is anti-extensive (so that id $\wedge \psi=\psi$ ). In that case

$$
\cdots \leq \psi^{n+1} \leq \psi^{n} \leq \psi^{n-1} \leq \cdots \leq \psi \leq i d .
$$

Define $\psi^{\infty}:=\bigwedge_{n \geq 1} \psi^{n}$. If $\psi^{\infty}$ is idempotent, or equivalently if $\psi \psi^{\infty}=\psi^{\infty}$, then $\psi^{\infty}$ is an opening, and we have $\check{\psi}=\psi^{\infty}$. Unfortunately, $\psi^{\infty}$ need not be idempotent. In [12] we have given the following counterexample. Let $\psi: \mathcal{P}(\mathbb{Z}) \rightarrow \mathcal{P}(\mathbb{Z})$ be the increasing, anti-extensive translation invariant operator given by $\psi(X)=(X \oplus A) \cap X$, where $A=$ $\{\ldots,-5,-3,-1,2\}$. Take $X=\{0,1,3,5, \ldots\}$; then, by a straightforward calculation, $\psi^{n}(X)=\{0,2 n+1,2 n+3,2 n+5, \ldots\}$ (see Fig. 7), and so $\psi^{\infty}(X)=\{0\}$. However, $\psi \psi^{\infty}(X)=\psi(\{0\})=\emptyset \neq \psi^{\infty}(X)$.

Let $X_{n}$ be a sequence in $\mathcal{L}$; we say that $X_{n} \downarrow X$ if $X_{n}$ is decreasing and $X=\bigwedge_{n \geq 1} X_{n}$. Similarly, we define $X_{n} \uparrow X$. An increasing operator $\psi$ on $\mathcal{L}$ is called $\downarrow$ - continuous if $X_{n} \downarrow X$ 


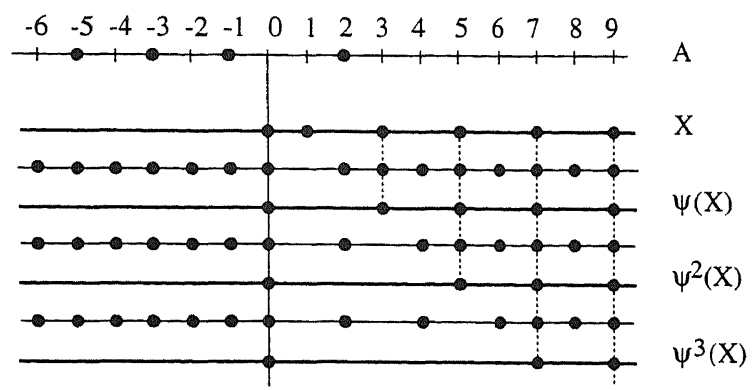

FIG. 7. An example of an anti-extensive operator $\psi$ for which $\psi \psi^{\infty} \neq \psi^{\infty}$.

implies that $\psi\left(X_{n}\right) \downarrow \psi(X)$. If the anti-extensive operator $\psi$ is $\downarrow$-continuous, then

$$
\psi \psi^{\infty}(X)=\psi\left(\bigwedge_{n \geq 1} \psi^{n}(X)\right)=\bigwedge_{n \geq 1} \psi^{n+1}(X)=\psi^{\infty}(X),
$$

meaning that $\breve{\psi}=\psi^{\infty}$; refer to [37] for examples.

We show how we can extend such arguments for operators which are not necessarily increasing; see Heijmans and Serra [20] for a comprehensive theory. For a sequence $X_{n}$ in $\mathcal{L}$ we define

$$
\begin{aligned}
& \liminf X_{n}=\bigvee_{N \geq 1} \bigwedge_{n \geq N} X_{n}, \\
& \limsup X_{n}=\bigwedge_{N \geq 1} \bigvee_{n \geq N} X_{n} .
\end{aligned}
$$

It is obvious that $\lim \inf X_{n} \leq \lim \sup X_{n}$. We say that $X_{n} \rightarrow X$ if $\lim \inf X_{n}=\lim \sup X_{n}=$ $X$. If $X_{n} \downarrow X$ or $X_{n} \uparrow X$ then $X_{n} \rightarrow X$. An operator $\psi$ on $\mathcal{L}$ is said to be $\downarrow$-continuous if $X_{n} \rightarrow X$ implies that limsup $\psi\left(X_{n}\right) \leq \psi(X)$, and $\uparrow$-continuous if $X_{n} \rightarrow X$ implies that $\lim \inf \psi\left(X_{n}\right) \geq \psi(X)$. The operator $\psi$ is called continuous if it is both $\downarrow$ - continuous and $\uparrow$ continuous. It is not difficult to show that for increasing operators this definition is consistent with the definition given above.

In [20] and [17] one can find a general account of $\downarrow$ - and $\uparrow$-continuous operators. We quote some of the main results.

- Every erosion is $\downarrow$-continuous and every dilation is $\uparrow$-continuous.

- Every automorphism is continuous.

- Let $\mathcal{L}$ have a negation, then $\psi$ is $\downarrow$-continuous iff $\psi^{*}$ is $\uparrow$-continuous.

- The infimum of an arbitrary collection of $\downarrow$-continuous operators is $\downarrow$-continuous.

- If $\mathcal{L}$ is atomic, then any finite supremum of $\downarrow$-continuous operators is $\downarrow$-continuous. (It is worthwhile to remark that in [20] a more general result is stated which applies also to the function lattice $\operatorname{Fun}(E, \overline{\mathbb{R}})$.)

Remark 4.4. The complete lattice $\mathcal{F}\left(\mathbb{R}^{d}\right)$ of closed subsets of $\mathbb{R}^{d}$ can be endowed with a topology which is based on the hit-or-miss operator of $\$ 2$. This topology is called the hitor-miss topology and has been investigated in great detail by Matheron [28]. In [20] we have pointed out the relation between the lattice convergence defined here and convergence in the sense of the hit-or-miss topology.

In $\S 2$ we have introduced the notion of a finite window operator in combination with translation invariance. We can easily extend this definition for operators which are not translation invariant. 
DefinITION 4.5. Let $E$ be an arbitrary set. The operator $\psi: \mathcal{P}(E) \rightarrow \mathcal{P}(E)$ is a finite window operator if for every $h \in E$ there exists a finite set $A(h) \subseteq E$ such that

$$
h \in \psi(X) \Longleftrightarrow h \in \psi\left(X \cap A^{\prime}\right)
$$

for $X \subseteq E$ and $A^{\prime} \supseteq A(h)$.

For translation invariant operators one can take $A(h)=A_{h}$. The proof of the following result can be found in [20] and also in [17].

PROPOSITION 4.6. Every finite window operator on $\mathcal{P}(E)$ is continuous.

One can show that finite unions, intersections, and compositions of finite window operators are finite window operators. Furthermore, $\psi$ is a finite window operator if and only if $\psi^{*}$ is a finite window operator.

For an arbitrary operator $\psi$ on $\mathcal{L}$ we define

$$
\psi^{\infty}(X)=\limsup \psi^{n}(X), \quad \psi_{\infty}(X)=\liminf \psi^{n}(X)
$$

If $\psi_{\infty}=\psi^{\infty}$, then we write $\psi^{n} \rightarrow \psi^{\infty}$.

If $\psi$ is anti-extensive, or more generally if $\psi^{2} \leq \psi$, then $\psi^{n+1} \leq \psi^{n}$, and in that case $\psi^{\infty}=\psi_{\infty}=\bigwedge_{n>1} \psi^{n}$. In [20] the following results have been established.

THEOREM 4.7. Let $\psi$ be an arbitrary operator on $\mathcal{L}$.

(a) If $\psi$ is anti-extensive and $\downarrow$-continuous then $\psi^{\infty}=\bigwedge_{n \geq 1} \psi^{n}$ is idempotent.

(b) If $\psi$ is extensive and $\uparrow$-continuous then $\psi^{\infty}=\bigvee_{n \geq 1} \psi^{n}$ is idempotent.

(c) If $\psi^{n} \rightarrow \psi^{\infty}$ and $\psi$ is continuous then $\psi^{\infty}$ is idempotent.

COROLLARY 4.8. Let $\psi$ be an increasing operator on $\mathcal{L}$.

(a) If $\psi$ is $\downarrow$-continuous then $\breve{\psi}=\wedge_{n \geq 1}(i d \wedge \psi)^{n}$.

(b) If $\psi$ is $\uparrow$-continuous then $\check{\psi}=\bigvee_{n \geq 1}(i d \vee \psi)^{n}$.

4.4. Construction of a filter which is self-dual. In [37] we have discussed some examples of openings generated by iteration. Here we discuss the construction of a morphological filter which utilizes Theorem 4.7(c). For $E$ we take the discrete hexagonal grid. Let $H$ be the discrete hexagon with radius one centered at the origin. Then $H$ contains seven points, the origin $a_{0}$ and its six neighbors, denoted by $a_{1}, \ldots, a_{6}$; see Fig. 8(a). Let $b$ be the Boolean threshold function (see Muroga [29] and van den Boomgaard [45]):

$$
b\left(x_{0}, x_{1}, \ldots, x_{6}\right)= \begin{cases}1, & \text { if } 3 x_{0}+x_{1}+x_{2}+\cdots+x_{6} \geq 5 \\ 0, & \text { otherwise }\end{cases}
$$

Let $\psi=\psi_{b}$ be given by (2.23):

$$
\psi(X)=\left\{h \in E \mid 3 X(h)+X\left(a_{1}+h\right)+\cdots+X\left(a_{6}+h\right) \geq 5\right\} .
$$

moment of reflection shows that this operator acts as follows: a point $h$ in the set $X$ lies the transformed set $\psi(X)$ if and only if at least two of its six hexagonal neighbors also lie ide $X$. If, say, only one neighbor lies in $X$ then $3 X(h)+X\left(a_{1}+h\right)+\cdots+X\left(A_{6}+h\right)=4$ 1 the threshold 5 is not reached. If $h$ lies in $X^{c}$ it is contained in $\psi(X)$ if at least five of its xagonal neighbors lie in $X$. In Fig. 8(b) $\psi_{b}$ changes the value of the central point, whereas 1 (c) this value remains unchanged.

In this procedure the background is treated analogously: a point in the background $X^{c}$ with at least two neighbors in $X^{c}$ stays in the background after transformation. In other words, the operator $\psi$ is self-dual. In fact, the self-duality of $\psi$ is a consequence of the self-duality of the underlying Boolean function $b$. Since $b$ is positive the operator $\psi$ is increasing. Furthermore, it is evident that $\psi$ is a finite window operator, and from Proposition 4.6 we conclude that it is 


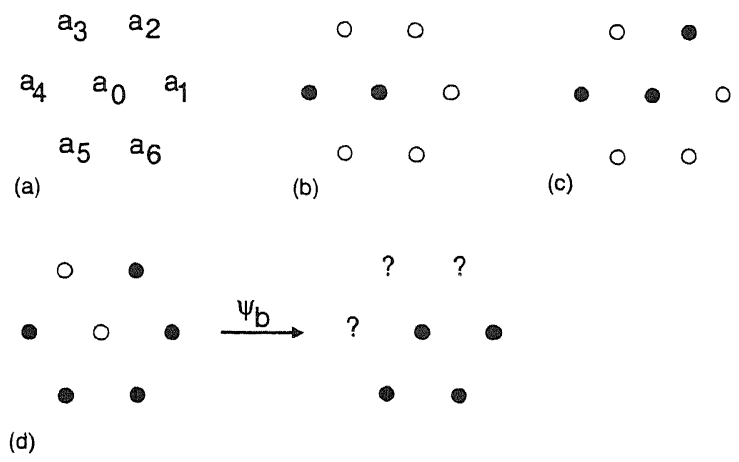

FIG. 8. See text.

continuous. We show that the sequence $\psi^{n}$ is convergent. This is an immediate consequence of the following nice property of $\psi$ : for every $X$ the sequence $X, \psi(X), \psi^{2}(X), \ldots$ is pointwise monotone. This means that for every point $h$ the value $\left[\psi^{n}(X)\right](h)$ (which is 0 or 1 ) can change at one instance at most. So it is either of the form $0,0, \ldots, 0,1,1,1, \ldots$ or of the opposite form. To see why this property holds, consider the configuration in Fig. 8(d). Here the central point changes its value from 0 to 1 ; however, its neighbors $a_{5}, a_{6}, a_{1}$ remain unchanged at this step. But the configuration formed by the points $a_{0}, a_{5}, a_{6}, a_{1}$ is stable under $\psi$, meaning that its values remain constant at subsequent iteration steps. We may now conclude that $\psi^{n} \rightarrow \psi^{\infty}$, where $\psi^{\infty}(X)$ is the pointwise limit under the iteration procedure described above. From Theorem 4.7(c) we conclude that $\psi^{\infty}$ is a filter. Since $\psi$ is self-dual, the filter $\psi^{\infty}$ is selfdual too.

\section{Geometrical aspects.}

5.1. Distance. The theory discussed so far may give the impression that mathematical morphology is a highly algebraic theory. This impression is correct to a limited extent only. As we pointed out in the introduction, the primary goal of morphology is to extract geometrical information from an image. For that matter topological and geometrical concepts are of paramount importance. In this section we illustrate this fact by means of two examples. First we introduce geodesic operators, a class of transformations which proved very useful for many different applications, including image segmentation. And second, we discuss granulometries which form the basis for the computation of size distributions. For both examples the notion of a metric plays an important role.

Throughout this section we assume that $E=\mathbb{R}^{d}$.

DEFINITION 5.1. A metric on $E$ is a function $d: E \times E \rightarrow \mathbb{R}_{+}$such that for $x, y, z \in E$,

$$
\begin{aligned}
& d(x, y)=0 \Longleftrightarrow x=y ; \\
& d(x, y)=d(y, x) ; \\
& d(x, z) \leq d(x, y)+d(y, z) .
\end{aligned}
$$

The latter property is called the triangle inequality. The metric $d$ is called a translation invariant metric if it satisfies the additional property

$$
d(x+h, y+h)=d(x, y)
$$

for $x, y, h \in E$. If

$$
d(r x, r y)=|r| d(x, y),
$$

for $r \in \mathbb{R}$, then $d$ is called homogeneous. 
Let $B(r)$ be the ball centered at 0 with radius $r \geq 0$,

$$
B(r)=\{x \in E \mid d(x, 0) \leq r\}
$$

A set $X \subseteq E$ is called symmetric if $X=\check{X}$, where $\check{X}=\{-x \mid x \in X\}$.

PROPOSITION 5.2. Let $d$ be a translation invariant metric; then the balls $B(r)$ have the following properties:

$$
\begin{aligned}
& B(0)=\{0\} \\
& B(r) \text { is symmetric and } 0 \in B(r) \\
& B(r) \oplus B(s) \subseteq B(r+s) \\
& B(r)=\bigcap_{r^{\prime}>r} B\left(r^{\prime}\right) .
\end{aligned}
$$

Proof. (B1) and (B4) are obvious. (B2) follows readily from the observation that

$$
d(x, 0)=d(x-x, 0-x)=d(0,-x)=d(-x, 0)
$$

To prove (B3) take $x \in B(r)$ and $y \in B(s)$. We show that $x+y \in B(r+s)$. Since $d(0, x) \leq r$ and $d(0, y)=d(0,-y) \leq s$ we get that $d(x,-y) \leq r+s$. Now our claim follows from the observation that

$$
d(x,-y)=d(x+y, 0)=d(0, x+y)
$$

On the other hand, the existence of a family of sets $B(r)$ which satisfy the axioms (B 1)(B4) implies the existence of a translation invariant metric $d$, namely

$$
d(x, y)=\inf \{r \geq 0 \mid x-y \in B(r)\} .
$$

5.2. Convexity. If $d$ is a homogeneous, translation invariant metric, then the function $p: E \rightarrow \mathbb{R}_{+}$given by

$$
p(x)=d(x, 0)
$$

defines a norm, and the unit ball with respect to this norm

$$
B=\{x \in E \mid p(x) \leq 1\}
$$

is convex. Furthermore, this set is compact with respect to the topology generated by this norm. Conversely, if $B$ is compact, convex, and symmetric, and if 0 is contained in the interior of $B$, then

$$
p_{B}(x)=\inf \{r>0 \mid x \in r B\}
$$

defines a norm on $E$. The function $p_{B}$ is called the Minkowskifunctional or gauge functional; see Valentine [44]. In this case the balls of radius $r$ are given by $B(r)=r B$. It is easy to check that

$$
r B \oplus s B=(r+s) B, \quad r, s \geq 0
$$

if $B$ is convex. In fact, the inclusion " $\supseteq$ " holds for any set $B$. One may wonder which sets $B$ "solve" equation (5.3). Is convexity required? Obviously, the set $B \subseteq \mathbb{R}^{2}$ consisting of the closed first and third quarter-plane satisfies the equation but is definitely not convex. A 
very general answer to the question has been given by Matheron [28]. Let $\mathcal{K}^{\prime}$ be the space of nonvoid compact subsets of $\mathbb{R}^{d}$ provided with the topology generated by the Hausdorff metric [9], [28].

THEOREM 5.3. Let $B(\cdot): \mathbb{R}_{+} \rightarrow \mathcal{K}^{\prime}$ be a continuous mapping. Then

$$
B(r) \oplus B(s)=B(r+s), \quad r, s \geq 0,
$$

if and only if $B(r)=r B$ for some compact, convex set $B$.

Let $A, X \subseteq E$. We say that $X$ is $A$-open if $X=X \circ A$, where the opening "o" is defined by (2.16). It is obvious that $B(s)$ is $B(r)$-open for $s \geq r$ if the semigroup property (5.4) holds. Furthermore, Matheron [28] has proved the following result.

THEOREM 5.4. Let $B \subseteq E$ be compact. Then $s B$ is $r B$-open for $s \geq r$ if and only if $B$ is convex.

As a corollary we get that $s B$ is $r B$-open $(s \geq r)$ if and only if the semigroup property (5.3) holds (given that $B$ is compact). This result cannot be extended to families $B(r)$ which are not obtained by scaling. In fact, introducing the notion of Stieltjes-Minkowski integral in the space $\mathcal{K}^{\prime}$, Matheron constructed a class of mappings $B: \mathbb{R}_{+} \rightarrow \mathcal{K}^{\prime}$ such that $B(s)$ is $B(r)$-open for $s \geq r$. As a special member of this class we mention

$$
B(r)= \begin{cases}B_{1}, & 0 \leq r \leq r_{1} \\ B_{1} \oplus B_{2}, & r>r_{1}\end{cases}
$$

Note that this example does not satisfy semigroup property (5.4).

5.3. Geodesic operators. The reason for this discussion on metrics and its relation to convexity becomes clear below where we treat granulometries. But first we introduce a class of metrics which is neither translation invariant nor homogeneous, but which is of great practical value. This class comprises the so-called geodesic metrics. Let $M \subseteq \mathbb{R}^{d}$ be a fixed set called the mask set or mask image. Let $x, y$ be two points inside $M$. If $x, y$ lie in the same (arcconnected) component of $M$ then there exist paths inside $M$ connecting $x$ and $y$; see Fig. 9 for an illustration.
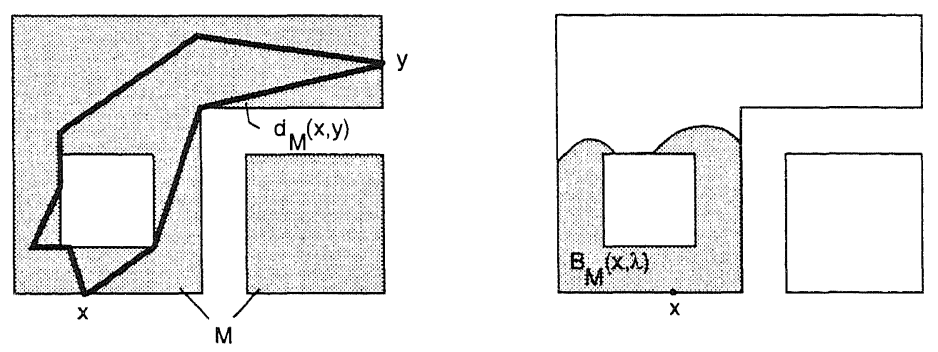

FIG. 9. Left: a mask image $M$ and two geodesic paths from $x$ to $y$. The path at the right is a geodesic path and its length is $d_{M}(x, y)$. Right: the geodesic ball $B_{M}(x, \lambda)$ centered at $x$ with radius $\lambda$. Note that $B_{M}(x, \lambda)$ has an empty intersection with the bottom-right component of $M$, for every $\lambda>0$.

The shortest of these paths is called a geodesic path, and its length is denoted by $d_{M}(x, y)$. We call this value the geodesic distance between $x$ and $y$. If $x$ and $y$ lie in different components of $M$ then we put $d_{M}(x, y)=\infty$. Since $d_{M}$ may assume the value $\infty$ it is not a metric in the classical sense. However, it is not difficult to show that $d_{M}$ satisfies the axioms (D1)-(D3). Geodesics have been studied in great detail by differential geometers. A nice treatment can be found in Busemann [5]. The metric $d_{M}$ is also called intrinsic metric or inner metric in the literature; see, e.g., Rinow [32]. 
Using geodesic distance we can build a class of transformations generally referred to as geodesic operators. Let $B_{M}(x, r)$ be the geodesic ball with center $x$ and radius $r$,

$$
B_{M}(x, r)=\left\{y \in M \mid d_{M}(x, y) \leq r\right\}
$$

see Fig. 9. by

On $\mathcal{P}(M)$ we define the geodesic dilations $\delta^{r}(\cdot \mid M)$ and erosions $\varepsilon^{r}(\cdot \mid M)$, respectively,

$$
\begin{aligned}
& \delta^{r}(X \mid M)=\bigcup_{x \in X} B_{M}(x, r) \\
& \varepsilon^{r}(X \mid M)=\left\{x \in X \mid B_{M}(x, r) \subseteq X\right\}
\end{aligned}
$$

It is easy to check that the pair $\left(\varepsilon^{r}(\cdot \mid M), \delta^{r}(\cdot \mid M)\right)$ forms an adjunction on $\mathcal{P}(M)$. Furthermore, these operators satisfy the semigroup relations

$$
\delta^{r}(\cdot \mid M) \delta^{s}(\cdot \mid M)=\delta^{r+s}(\cdot \mid M), \quad \varepsilon^{r}(\cdot \mid M) \varepsilon^{s}(\cdot \mid M)=\varepsilon^{r+s}(\cdot \mid M)
$$

refer to $[17, \S 9.5]$.

As an application of the geodesic approach we mention the "skeleton by influence zones," usually referred to as the SKIZ. Suppose that the set $X \subseteq M$ comprises $n$ objects, $X=$ $X_{1} \cup X_{2} \cup \ldots \cup X_{n}$, which have empty intersection. A point $h \in M$ is said to belong to the influence zone of the object $X_{k}$ if $d_{M}\left(h, X_{k}\right)<d_{M}\left(h, X_{l}\right)$ for $l \neq k$. Here $d_{M}\left(h, X_{k}\right)$ is defined as the length of the shortest geodesic path from $h$ to some point of $X_{k}$. The SKIZ is defined as the collection of boundaries which separate the influence zones. It is often used for segmentation problems. Consult [24] and [40] for more information.

A geodesic operator which turns out to be of great value in practice is the geodesic reconstruction, defined by

$$
\rho(X \mid M)=\bigcup_{r>0} \delta^{r}(X \mid M)
$$

Since a union of dilations is a dilation (Proposition 3.4) we get that $\rho(\cdot \mid M)$ is a dilation. It extracts the connected components of $M$ which have nonempty intersection with $X$; see Fig. 10.

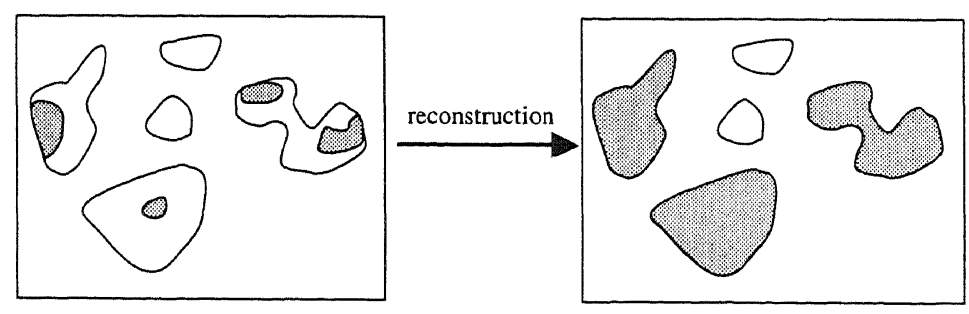

FIG. 10. Geodesic reconstruction.

Here we mention one application of the reconstruction $\rho(\cdot \mid M)$. Suppose we apply some opening $\alpha$ to an image with the intention to remove small noise particles. This opening may also affect larger particles, in particular their contours. We can neutralize this effect by taking the reconstruction of $\alpha(X)$ inside the mask set $X$. That is, we define

$$
\check{\alpha}(X)=\rho(\alpha(X) \mid X) .
$$


One can easily show that $\check{\alpha}$ is again an opening. Furthermore, this procedure to modify openings preserves invariance properties. For instance, if $\alpha$ is translation invariant, then $\check{\alpha}$ is such as well.

5.4. Granulometries. A granulometry on $\mathcal{P}(E)$ is a one-parameter family $\left\{\alpha_{r} \mid r>0\right\}$ of openings on $\mathcal{P}(E)$ such that

$$
\alpha_{r} \alpha_{s}=\alpha_{s} \alpha_{r}=\alpha_{s}, \quad s \geq r,
$$

or equivalently,

$$
\alpha_{s} \leq \alpha_{r}, \quad s \geq r .
$$

The proof that (5.11) and (5.12) are equivalent is left as an exercise to the reader. Granulometries are used in practice to obtain size distributions. If $m$ denotes Lebesgue measure them $m\left(\alpha_{r}(X)\right)$ can be interpreted as the total volume of particles with size $\geq r$.

If every opening $\alpha_{r}$ is a structural opening (cf. $\left.\S 2\right)$ then we say that $\left\{\alpha_{r}\right\}$ is a structural granulometry. If every opening $\alpha_{r}$ is translation invariant then we speak of a translation invariant granulometry. The following observation is of great importance with respect to translation invariant granulometries: if $B$ is $A$-open, that is, if $B \circ A=B$, then $X \circ B \subseteq X \circ A$ for every set $X \subseteq E$. If $B(r)$ is a family of subsets of $E$ which satisfy the semigroup property $B(r) \oplus B(s)=B(r+s)$, then the family $\alpha_{r}$ given by $\alpha_{r}(X)=X \circ B(r)$ defines a structural translation invariant granulometry. Namely, if the semigroup relation holds then $B(s)$ is $B(r)$-open if $s \geq r$, meaning that $\alpha_{s} \leq \alpha_{r}$.

If $\left\{\alpha_{r}\right\}$ is a translation invariant granulometry which is scale-compatible in the sense that

$$
\alpha_{r}(X)=r \alpha_{1}\left(r^{-1} X\right) \text { for every } r>0 \text { and } X \subseteq E,
$$

then $\left\{\alpha_{r}\right\}$ is called a Minkowski granulometry. Note that Matheron [28] and Serra [40] call such granulometries Euclidean granulometries.

It is apparent that condition (5.13) is equivalent to

$$
\operatorname{Inv}\left(\alpha_{r}\right)=r \operatorname{Inv}\left(\alpha_{1}\right)
$$

In fact, if $\left\{\alpha_{r}\right\}$ is a Minkowski granulometry then $\operatorname{Inv}\left(\alpha_{1}\right)$ is closed under translations and multiplications with scalars $r \geq 1$.

THEOREM 5.5. Let $\left\{\alpha_{r}\right\}$ be a Minkowski granulometry; then there is a family $\mathcal{B} \subseteq \mathcal{P}\left(\mathbb{R}^{d}\right)$ such that

$$
\alpha_{r}(X)=\bigcup_{s \geq r} \bigcup_{B \in \mathcal{B}} X \circ s B .
$$

Conversely, if $\mathcal{B} \subseteq \mathcal{P}\left(\mathbb{R}^{d}\right)$, then $\alpha_{r}$ given by (5.15) defines a Minkowski granulometry.

Of particular interest are structural Minkowski granulometries. From the considerations above it is easy to deduce that the openings in such a granulometry are of the form $\alpha_{r}(X)=$ $X \circ r B$. In order that $s B$ is $r B$-open for $s \geq r$ it is necessary and sufficient that $B$ be convex (that is, if we presume compactness on $B$ ); cf. Theorem 5.4.

We emphasize that a granulometry is a metric concept. In fact. we have seen that a structural Minkowski granulometry is characterized by a unique, compact, convex set, and therefore by a homogeneous, translation invariant metric. (To make this precise, one has to impose some extra conditions which guarantee that the convex set which defines the granulometry is compact and has nonempty interior, but we shall not deal with these technicalities here.) If $\left\{\delta^{r} \mid r>0\right\}$ is a family of dilations on $\mathcal{P}(E)$ which satisfy the semigroup property

$$
\delta^{r} \delta^{s}=\delta^{r+s}
$$


(see also (5.8)), and if $\varepsilon^{r}$ is the erosion related to $\delta^{r}$ by adjunction, then the openings $\alpha_{r}=\delta^{r} \varepsilon^{r}$ define a granulometry. This observation holds for arbitrary complete lattices, and can, e.g., be used as a basis for a theory of granulometries on discrete spaces where the notion of convexity is rather cumbersome. Below we shall meet a class of dilations for which condition (5.16) holds.

5.5. Metric dilations. If there is a real number $L>0$ such that $d(x, y) \leq L$ for all $x$, $y \in X$ then the set $X$ is called bounded. The following definitions stem from Busemann [5].

DEFINITION 5.6. The metric space $(E, d)$ is finitely compact if each bounded subset comprising infinitely many points contains at least one accumulation point.

Note that $\mathbb{R}^{d}$ endowed with the Euclidean metric is finitely compact.

Given three distinct points $u, x, y \in E$, we say that $u$ lies between $x$ and $y$ if $d(x, u)+$ $d(u, y)=d(x, y)$. In the Euclidean metric a point lies between $x$ and $y$ iff it is located on the straight line segment between $x$ and $y$.

DEFINITION 5.7. A subset $X$ of a metric space $E$ is called metrically convex or M-convex if for every pair of distinct points there exists a point located between them. If for every two such points there lies a geodesic path between them then the set X is called continuously Mconvex.

It is easy to see that every continuously $M$-convex space which has an intrinsic metric is M-convex. The converse, however, is in general not true. by

Consider a metric space $(E, d)$. For every $r \geq 0$ we define the metric dilation $\delta^{r}$ on $\mathcal{P}(E)$

$$
\delta^{r}(X)=\bigcup_{x \in X} B(x, r)
$$

cf. (5.6). It is easy to prove that the family $\delta^{r}, r \geq 0$, obeys the following properties (for $x, y \in E)$ :

$$
\begin{aligned}
& \delta^{0}=\mathrm{id} ; \\
& \delta^{s} \geq \delta^{r} \quad \text { if } s \geq r \quad \text { and } \bigcap_{s>r} \delta^{s}(\{x\})=\delta^{r}(\{x\}) ; \\
& x \in \delta^{r}(\{y\}) \Longleftrightarrow y \in \delta^{r}(\{x\}) ; \\
& \delta^{r} \delta^{s} \leq \delta^{r+s}, \quad r, s \geq 0 ; \\
& \bigcup_{r \geq 0} \delta^{r}(\{x\})=E .
\end{aligned}
$$

If the dilations $\delta^{r}$ are given, one can recover the distance $d$ as follows:

$$
d(x, y)=\inf \left\{r>0 \mid y \in \delta^{r}(\{x\})\right\} .
$$

In fact, there is a one-one correspondence between distance functions $d$ and metric dilations $\left\{\delta^{r} \mid r \geq 0\right\}$ satisfying $(\Delta 0)-(\Delta 4)$.

THEOREM 5.8. If $E$ is continuously $M$-convex then the metric dilations satisfy

$$
\delta^{r} \delta^{s}=\delta^{r+s}, \quad r, s \geq 0
$$

-onversely, if $(E, d)$ is finitely compact and if the semigroup relation (5.19) holds then $(E, d)$ $s$ continuously $M$-convex.

A proof of this result can be found in [17].

5.6. Distance transform. For $h \in E$ and $X \subseteq E$ the distance $d(h, X)$ is defined by

$$
d(h, X)=\inf _{x \in X} d(h, x)
$$


The distance transform $\Delta(X)$ of a set $X$ is

$$
[\Delta(X)](h)=d(h, X), \quad h \in E .
$$

We write $\Delta(X, h)$ instead of $[\Delta(X)](h)$. An example is depicted in Fig. 11 .

In lattice theoretical terms the distance transform is a decreasing operator from $\mathcal{P}(E)$ to $\mathcal{H}=\operatorname{Fun}\left(E, \overline{\mathbb{R}}_{+}\right)$, the functions from $E$ to $\overline{\mathbb{R}}_{+}=[0, \infty]$. There exists the following simple relation between the distance transform and the metric dilations.
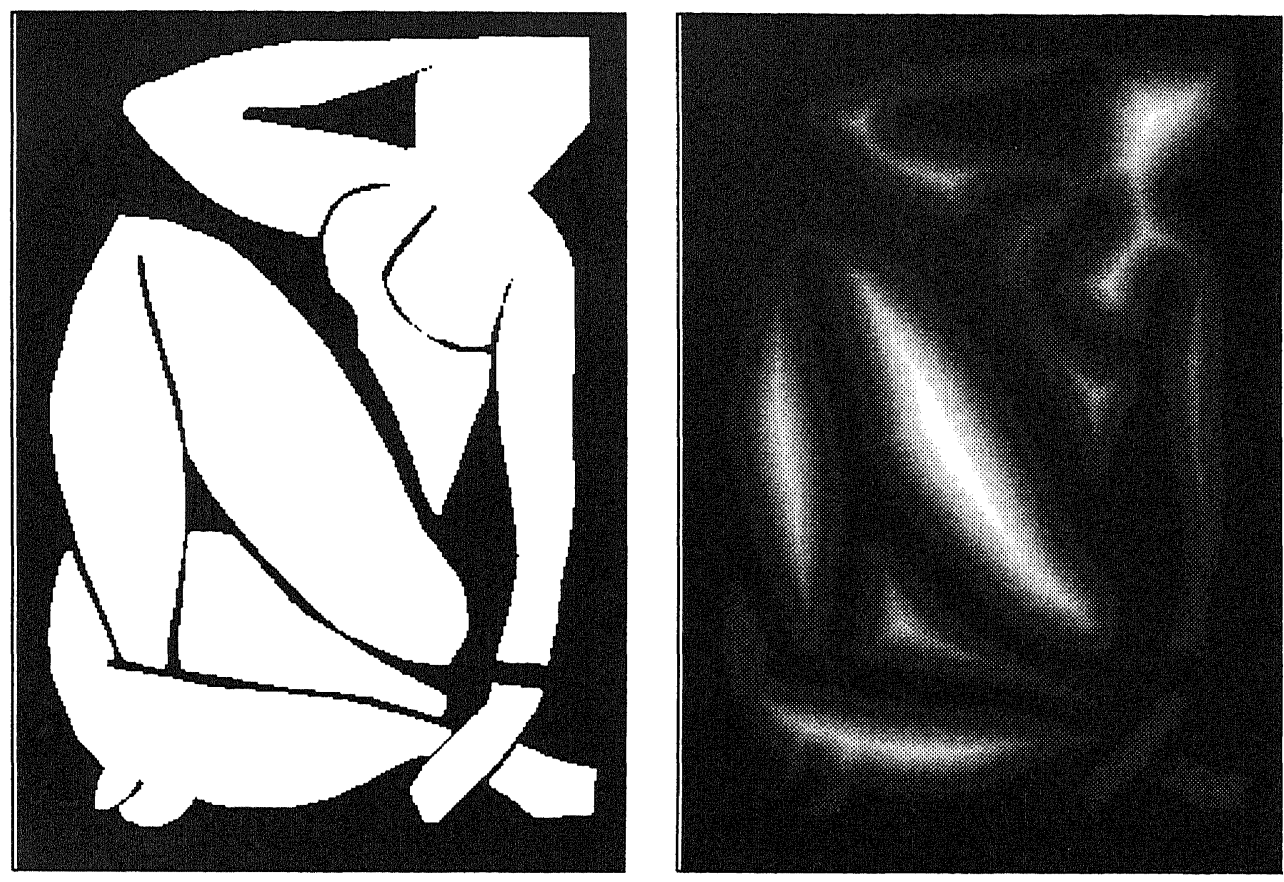

FIG. 11. A set $X$ (H. Matisse's "Nu Bleu III", 1952) and its distance transform $\Delta(X)$ shown as a grey-scale image: the higher the value $\Delta(X, h)$, the brighter the image at the point $h$.

PROPOSITION 5.9. Assume that $(E, d)$ is a finitely compact metric space and that $X$ is a closed subset of E. Then

$$
\Delta(X, h)=\inf \left\{r \geq 0 \mid h \in \delta^{r}(X)\right\},
$$

and

$$
\delta^{r}(X)=\{h \in E \mid \Delta(X, h) \leq r\}
$$

Define the operator $\Delta^{\leftarrow}: \mathcal{H} \rightarrow \mathcal{P}(E)$ by

$$
\Delta^{\leftarrow}(F)=\left[\bigcup_{h \in E} B^{\circ}(h, F(h))\right]^{c},
$$

where $B^{\circ}(x, r)$ is the open ball with radius $r$ centered at $x$. The following result was stated in [17, Prop. 9.41].

PROPOSITION 5.10. If $(E, d)$ is finitely compact, then the pair of operators $\left(\Delta^{\leftarrow}, \Delta\right)$ constitutes an adjunction between $\mathcal{H}^{\prime}$ (the opposite lattice of $\mathcal{H}$ ) and $\mathcal{P}(E)$. The closing $\Delta^{-} \Delta$ on $\mathcal{P}(E)$ maps a set $X$ onto its closure $\bar{X}$. 
5.7. Metrics and cost functions. In [17] (see also [37]) we have described a systematic method to construct metrics and their corresponding distance transform; here we will give the main ingredients of this construction.

DEFINITION 5.11. A cost function is a function $c: E \times E \rightarrow \overline{\mathbb{R}}_{+}$which satisfies the first two properties of a metric:

$$
\begin{gathered}
c(x, y)=0 \quad \text { if and only if } x=y \\
c(x, y)=c(y, x) \text { for } x, y \in E .
\end{gathered}
$$

If $c$ is a cost function, then the operator

$$
\mathcal{E}_{c}(F)(x)=\bigwedge_{h \in E}[F(h)+c(h, x)]
$$

defines an erosion on $\mathcal{H}$. This erosion is anti-extensive, i.e., $\mathcal{E}_{c}(F) \leq F$. It is also easy to see that

$$
\mathcal{E}_{\bigwedge_{i \in I} c_{i}}=\bigwedge_{i \in I} \mathcal{E}_{\mathcal{c}_{i}}
$$

:or every collection $c_{i}, i \in I$. Let, for $X \subseteq E, 0_{X}$ be the function which is 0 on $X$ and $\infty$ on he complement $X^{c}$. If $x \in E$ then we write $\mathbf{0}_{x}$ instead of $\mathbf{0}_{\{x\}}$. It is obvious that

$$
c(x, y)=\mathcal{E}_{c}\left(\mathbf{0}_{x}\right)(y) .
$$

If $d$ is a metric then the distance transform can be expressed in terms of $\mathcal{E}_{d}$ :

$$
\Delta(X)=\mathcal{E}_{d}\left(0_{X}\right) .
$$

We define the convolution of two cost functions $c_{1}, c_{2}$ by

$$
\left(c_{1} * c_{2}\right)(x, y)=\bigwedge_{z \in E}\left[c_{1}(x, z)+c_{2}(z, y)\right] .
$$

One can easily show that

$$
\mathcal{E}_{c_{1} * c_{2}}=\mathcal{E}_{c_{2}} \mathcal{E}_{c_{1}}
$$

We define $c^{* n}$ recursively by

$$
\left\{\begin{array}{l}
c^{* 1}=c \\
c^{*(n+1)}=c * c^{* n}, \quad n \geq 1
\end{array}\right.
$$

It is easy to show that the sequence $c^{* n}$ is decreasing, that is,

$$
\cdots \leq c^{*(n+1)} \leq c^{* n} \leq c^{*(n-1)} \leq \cdots \leq c .
$$

Furthermore,

$$
\mathcal{E}_{c^{* n}}=\mathcal{E}_{c}^{n}, \quad n \geq 1 .
$$

PROPOSITION 5.12. Assume that $c$ is a cost function. The following assertions are equivalent:

(i) $c$ is a metric;

(ii) $c * c=c$;

(iii) $\mathcal{E}_{c}$ is idempotent. 
A proof can be found in [17]. Defining

$$
c^{* \infty}=\bigwedge_{n \geq 1} c^{* n},
$$

we derive from (5.20) and (5.23) that

$$
\mathcal{E}_{c^{* \infty}}=\mathcal{E}_{\bigwedge_{n \geq c^{* n}}}=\bigwedge_{n \geq 1} \mathcal{E}_{c^{* n}}=\bigwedge_{n \geq 1} \mathcal{E}_{c}^{n} .
$$

Since $\mathcal{E}_{c}$ is an erosion it distributes over infima, hence

$$
\mathcal{E}_{c * c^{* \infty}}=\mathcal{E}_{c} \mathcal{E}_{c^{*} \infty}=\mathcal{E}_{c}\left(\bigwedge_{n \geq 1} \mathcal{E}_{c}^{n}\right)=\bigwedge_{n \geq 1} \mathcal{E}_{c}^{n+1}=\mathcal{E}_{c^{* \infty}}
$$

here we have also used (5.22). This gives $c * c^{* \infty}=c^{* \infty}$, and thus we obtain $c^{* n} * c^{* \infty}=c^{* \infty}$ for $n \geq 1$. Taking the infimum over all $n \geq 1$ leads to

$$
c^{* \infty} * c^{* \infty}=c^{* \infty}
$$

To conclude that $c^{* \infty}$ is a metric we must be able to show that property (D1) holds, that is, $c^{* \infty}(x, y)=0$ iff $x=y$. The following simple example shows that this condition may be false. Let $E=\mathbb{Z}$ and $c(x, y)=e^{-|x-y|}$. It is evident that $(c * c)(x, y)=0$ everywhere. To exclude such pathological cases we assume that $c(x, y) \geq d(x, y)$ for some metric $d$ (e.g., the metric which is constantly $d_{0}$ if $x \neq y$ and 0 if $x=y$ ). We summarize our findings in the following theorem.

THEOREM 5.13. Let $c$ be a cost function and $d$ a metric such that $c(x, y) \geq d(x, y)$. Then $c^{* \infty}=\wedge_{n \geq 1} c^{* n}$ is a metric with $c^{* \infty} \geq d$. The corresponding erosion is given by

$$
\mathcal{E}_{c^{* \infty}}=\bigwedge_{n \geq 1} \mathcal{E}_{c}^{n}
$$

In the discrete case $E=\mathbb{Z}^{2}$, this procedure is often used to construct a metric which approximates the Euclidean metric. If $c$ is a translation invariant cost function on $\mathbb{Z}^{2}$, then $c$ is completely determined by the entries $c(0, h)$. In practice one considers cost functions with bounded support (taking the value $\infty$ outside the support); see Fig. 12 for some examples.
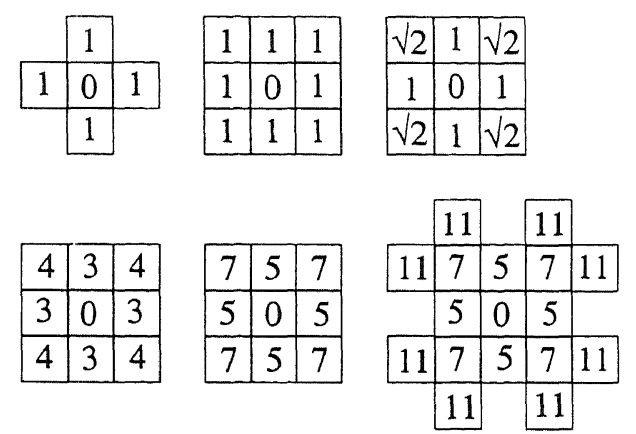

FlG. 12. Some chamfer cost functions used for the computation of a discrete metric on $\mathbb{Z}^{2}$.

The metrics resulting from such cost functions are called chamfer metrics; see Borgefors [3], [4]. Additional results about the utilization of chamfer metrics in mathematical morphology were derived by Nacken [30], [31]. 


\section{From sets to functions.}

6.1. Introduction. We have seen that, though morphology was originally developed for binary images, it can be extended to arbitrary complete lattices. In this section we consider lattices of functions which constitute representations of spaces of grey-scale images. There exist several, closely related, approaches to grey-scale morphology. A comprehensive discussion can be found in [17]. Other references include [14], [27], [40], and [43].

Before we start our discussion we point out that in this paper we restrict ourselves to the case where the grey-value set is $\overline{\mathbb{R}}=\mathbb{R} \cup\{-\infty, \infty\}$. However, many other choices are possible, such as $\overline{\mathbb{Z}}=\mathbb{Z} \cup\{-\infty, \infty\}$ or the finite set $\{0,1, \ldots, N\}$. A comprehensive discussion on morphological operators for image sequences can be found in [8].

Again, let $E=\mathbb{R}^{d}$ or $\mathbb{Z}^{d}$, and denote by $\operatorname{Fun}(E)$ the space of functions mapping $E$ into $\overline{\mathbb{R}}$. In $\S 3$ we have seen that $\operatorname{Fun}(E)$ is a complete lattice. Elements of Fun $(E)$ are denoted by $F, G$, etc. We can define a horizontal or spatial translation along $h \in E$ by

$$
F_{h}(x)=F(x-h), \quad x \in E,
$$

and a vertical or grey-scale translation along $v \in \mathbb{R}$ by

$$
(F+v)(x)=F(x)+v, \quad x \in E
$$

An operator $\Psi$ on Fun $(E)$ is called an $H$-operator if it is invariant under horizontal translations,

$$
\Psi\left(F_{h}\right)=[\Psi(F)]_{h}, \quad F \in \operatorname{Fun}(E), \quad h \in E,
$$

and a T-operator if it is invariant under both types of translations,

$$
\Psi\left(F_{h}+v\right)=[\Psi(F)]_{h}+v, \quad F \in \operatorname{Fun}(E), \quad h \in E, \quad v \in \mathbb{R}
$$

The set Fun $(E)$ is a complete lattice under the pointwise supremum (denoted by " $\bigvee$ ") and infimum (denoted " $\wedge$ ”). The mapping $F \rightarrow-F$ defines a negation (cf. $\S 3$ ). The negative operator of $\Psi$, denoted by $\Psi^{*}$, is defined by

$$
\Psi^{*}(F)=-\Psi(-F)
$$

For $F, G \in \operatorname{Fun}(E)$ we can define their Minkowski sum and difference, respectively, by

$$
(F \oplus G)(x)=\bigvee_{h \in E}[F(x-h)+G(h)]
$$

and

$$
(F \ominus G)(x)=\bigwedge_{h \in E}[F(x+h)-G(h)]
$$

In fact, these two operations result if one applies Proposition 3.7 to the complete lattice $\mathcal{L}=$ Fun $(E)$, with $\mathbb{T}$ the group of horizontal and vertical translations; refer, for missing details, to [17] and [37]. In our terminology, $\Delta_{G}(F)=F \oplus G$ defines a T-dilation and $\mathcal{E}_{G}(F)=F \ominus G$ a T-erosion. From the abstract theory (Proposition 3.2) it follows that $\Delta_{G}$ commutes with suprema and that $\mathcal{E}_{G}$ commutes with infima. By composing (or, alternatively, taking suprema and infima of) these two operators we can build openings, closings, alternating sequential filters, and many other grey-scale operators. 
6.2. Umbras. To visualize such grey-scale operators the notion of an umbra turns out to be very useful. Here we only sketch the idea; a comprehensive discussion can be found in [6], [16], and [17]. Let us, however, caution the mathematical reader that the umbra approach has always given rise to a lot of confusion and, even worse, false statements.

A set $U \subseteq E \times \mathbb{R}$ is called an umbra if

$$
(x, t) \in U \Longleftrightarrow(x, s) \in U \quad \text { for } s<t .
$$

If $F$ is a function then $U(F)=\{(x, t) \mid F(x) \geq t\}$ constitutes an umbra. To transform a function, one can alternatively transform the corresponding umbra. The idea is illustrated in Fig. 13 below.
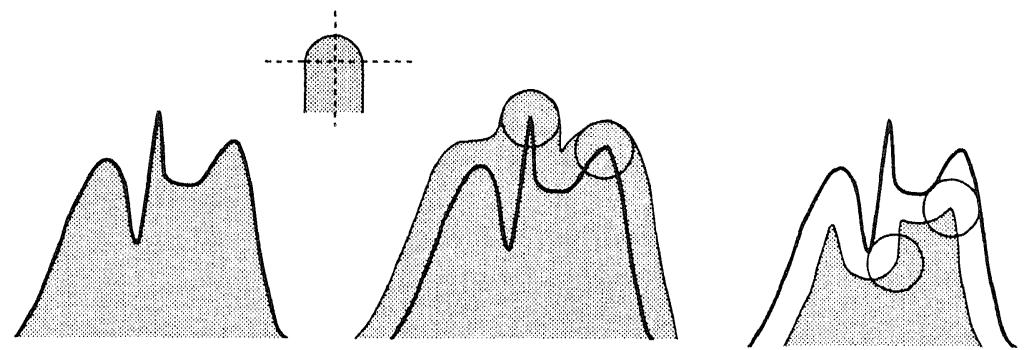

FIG. 13. Visualization of $F \oplus B$ and $F \ominus B$, where $B$ is a disk, by means of the umbra transform. Note that we may replace $B$ by the smallest umbra containing $B$.

A useful operator in mathematical morphology is the so-called top-hat transform which can be used for the extraction of narrow peaks.

We take an arbitrary grey-scale image $F$ and compute the opening $F \circ B=(F \ominus B) \oplus B$, where $B$ is the disk depicted in Fig. 14. This opening is usually referred to as the rolling-ball opening [43]. Then $F \circ B \leq F$. The top-hat transform is the difference $F-(F \circ B)$; see Fig. 14.
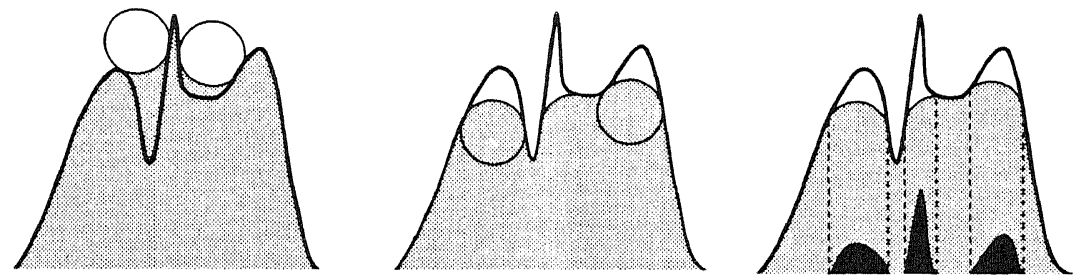

FIG. 14. Rolling-ball closing and opening and the top-hat transform.

Of particular interest in applications is the case where the structuring function $G$ in (6.2)(6.3) is flat; to be specific, $G(x)=0$ if $x \in A$ and $-\infty$ elsewhere; here $A \subseteq E$ is the domain of $G$. We derive the following expressions for $F \oplus G$ and $F \ominus G$ in this case:

$$
F \oplus G=\bigvee_{h \in A} F_{h}, \quad F \ominus G=\bigwedge_{h \in A} F_{-h}
$$

In Fig. 15 both operations are visualized with the aid of the umbra transform. We refer to the operators in (6.5) as flat operators; see also $\S 6.4$ below. 

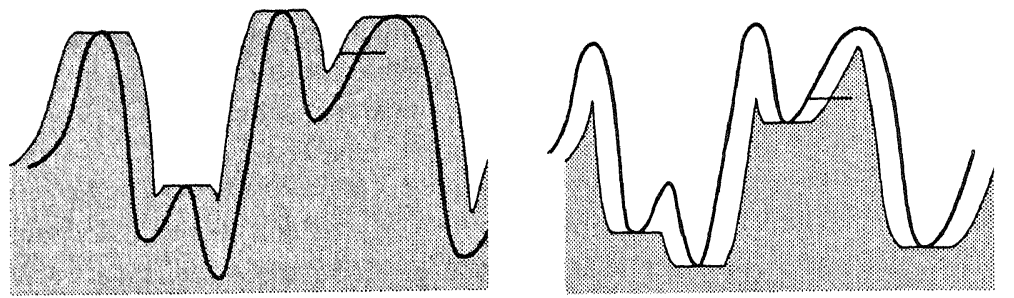

FIG. 15. Flat erosion $\mathcal{E}_{A}$ and flat dilation $\Delta_{A}$.

As an illustration of the use of flat structuring elements we mention the so-called morphological gradient. Recall that for a continuously differentiable function on $\mathbb{R}^{d}$ the gradient $\nabla F$ is defined as the $d$-vector $\left(\frac{\partial F}{\partial x_{1}}, \frac{\partial F}{\partial x_{2}}, \ldots, \frac{\partial F}{\partial x_{d}}\right)$. The morphological gradient is defined by

$$
\operatorname{grad}(F)=\lim _{r \downarrow 0} \frac{1}{2 r}[(F \oplus r D)-(F \ominus r D),
$$

where $D$ is the $d$-dimensional ball with radius 1 . It is easy to show that

$$
\operatorname{grad}(F)=\|\nabla F\|
$$

if $F$ is continuously differentiable. For a discrete image one defines

$$
\operatorname{grad}(F)=\frac{1}{2}[(F \oplus D)-(F \ominus D)]
$$

where $D$ is the discrete analogue of the unit ball. In the two-dimensional case one usually takes for $D$ the square consisting of nine points. In Fig. 16 we have computed the discrete gradient for a specific image.
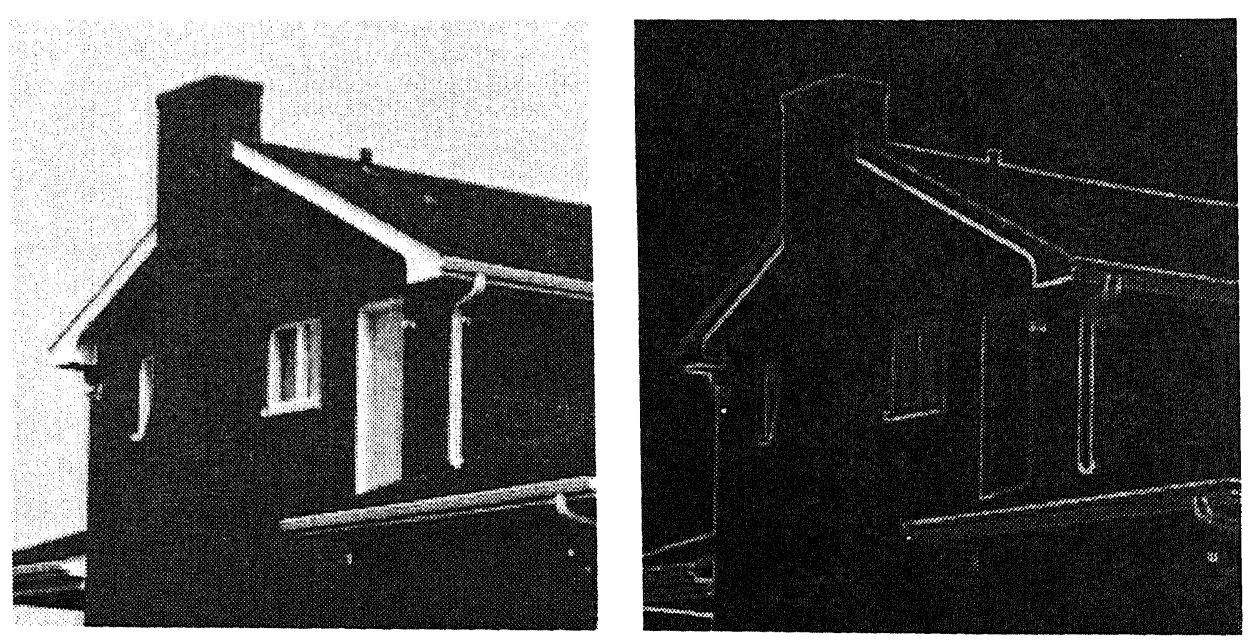

FIG. 16. Morphological gradient.

6.3. Thresholding. The grey-scale operators given above have been introduced without reference to the binary case. It is obvious, however, that these operators are closely related. In fact, both classes are particular examples of the complete lattice framework described in 
$\S 3$. We now present an alternative way to construct grey-scale morphological operators. The basic idea is to threshold a function at each grey-value $t$ (in other words, to take horizontal cross sections of the umbra), to apply a fixed increasing binary operator at every level (the resulting sets constitute again an umbra), and to compute the new function from the transformed threshold sets. This approach can be formalized if one returns to the lattice framework. This has the advantage that it applies to various grey-value sets, as well as to other function spaces, such as upper semi-continuous functions, a class of functions which has proved most relevant in the context of mathematical morphology. A comprehensive discussion can be found in [17, Chaps. 10, 11].

Let $\mathcal{L}$ be a complete lattice. We define $\mathcal{L} \diamond \overline{\mathbb{R}}$ as the space of all mappings $\mathrm{X}: \overline{\mathbb{R}} \rightarrow \mathcal{L}$ which are decreasing, and $\mathcal{L} \Delta \overline{\mathbb{R}}$ as the subset of mappings which satisfy

$$
\mathbf{X}\left(\bigvee_{i \in I} t_{i}\right)=\bigwedge_{i \in I} \mathbf{X}\left(t_{i}\right)
$$

for every family $\left\{t_{i} \mid i \in I\right\}$ in $\overline{\mathbb{R}}$ (such mappings are sometimes called anti-dilations). Define the operator $\hat{\mathbf{I}}: \mathcal{L} \diamond \overline{\mathbb{R}} \rightarrow \mathcal{L} \Delta \overline{\mathbb{R}}$ by

$$
(\hat{\mathbf{I X}})(t)=\bigwedge_{s<t} \mathbf{X}(s) .
$$

Then the mapping $\hat{\mathbf{I}}$ is a closing because it is increasing, idempotent, and satisfies $(\hat{\mathbf{I} X})(t) \geq$ $\mathbf{X}(t)$ for every $\mathbf{X} \in \mathcal{L} \diamond \overline{\mathbb{R}}$ and $t \in \overline{\mathbb{R}}$. The space $\mathcal{L} \Delta \overline{\mathbb{R}}$ is a complete lattice with the pointwise infimum of $\mathcal{L}$ and with supremum given by $\hat{\mathbf{I}}\left(\bigvee_{i \in I} \mathbf{X}_{i}\right)$ for any collection $\left\{\mathbf{X}_{i} \mid i \in I\right\}$ in $\mathcal{L} \Delta \overline{\mathbb{R}}$; here " $\bigvee$ " denotes the supremum in $\mathcal{L}^{\overline{\mathbb{R}}}$.

For a function $F: E \rightarrow \overline{\mathbb{R}}$ we define its threshold set $\mathbf{X}(F, t)$ by

$$
\mathbf{X}(F, t)=\{x \in E \mid F(x) \geq t\} .
$$

This mapping defines an isomorphism between Fun $(E)$ and $\mathcal{P}(E) \Delta \overline{\mathbb{R}}$ with inverse given by

$$
\mathbf{F}(\mathbf{X})(x)=\bigvee\{t \in \overline{\mathbb{R}} \mid x \in \mathbf{X}(t)\}
$$

Remark 6.1. If $E$ is a topological space, e.g., $\mathbb{R}^{d}$, and $\mathcal{F}(E)$ is the complete lattice of closed subsets of $E$, then $\mathcal{F}(E) \triangle \overline{\mathbb{R}}$ is isomorphic with the lattice of upper semi-continuous (u.s.c.) functions on $E$. Recall that a function $F$ is u.s.c. if for every $t \in \overline{\mathbb{R}}$ and $x \in E$ such that $t>F(x)$ there exists a neighborhood $V$ of $x$ such that $t>F(y)$ for $y \in V$. One can easily show that a function $F$ is u.s.c. if and only if every threshold set $\mathbf{X}(F, t)$ is closed.

6.4. Flat operators. Using the representation of $\operatorname{Fun}(E)$ given above it is easy to extend an increasing operator $\psi$ on $\mathcal{P}(E)$ to Fun $(E)$. Namely, we can represent a function $F$ by its threshold sets $\mathbf{X}(F, t)$. Applying $\psi$ to any such set gives a family of sets $\psi(\mathbf{X}(F, t))$ which is decreasing with respect to $t$, but which does not necessarily lie inside $\mathcal{P}(E) \triangle \overline{\mathbb{R}}$. To achieve this, we apply $\hat{\mathbf{I}}$ to this family. This gives an element of $\mathcal{P}(E) \Delta \overline{\mathbb{R}}$ and hence an element $\Psi(F)$ of $\operatorname{Fun}(E)$. It is obvious that the following relation holds:

$$
\mathbf{X}(\Psi(F), t)=\bigwedge_{s<t} \psi(\mathbf{X}(F, s))
$$

or alternatively,

$$
\Psi(F)(x)=\bigvee\{t \in \overline{\mathbb{R}} \mid x \in \psi(\mathbf{X}(F, t))\}
$$


We call $\Psi$ the flat function operator generated by $\psi$. The reader can easily verify that this construction fails if $\psi$ is not increasing. It can be shown that the given construction of flat operators is compatible with the formation of suprema, infima, compositions, and negation. The latter means, for example, that if $\psi$ is the generator of $\Psi$, then $\psi^{*}$ is the generator of $\Psi^{*}$. Furthermore, if $\psi$ is a dilation (erosion, closing, opening) then $\Psi$ is a dilation (erosion, closing, opening) as well. If we take, for example, $\psi$ to be the Minkowski set addition $\psi(X)=X \oplus A$ as given by (2.6), then $\Psi(F)=F \oplus G$ where $G$ is the flat function with domain $A$; see (6.5).

We briefly discuss the class of flat function operators associated with the finite window operators on $\mathcal{P}\left(\mathbb{Z}^{d}\right)$. In $\S 2$ we have seen that every such operator is of the form

$$
\psi_{b}(X)=\left\{h \in \mathbb{Z}^{d} \mid b\left(X\left(a_{1}+h\right), X\left(a_{2}+h\right), \ldots, X\left(a_{n}+h\right)\right)=1\right\},
$$

where $A=\left\{a_{1}, a_{2}, \ldots, a_{n}\right\}$ is a structuring element and $b$ a Boolean function. The operator $\psi_{b}$ is increasing if and only if $b$ is positive. Let $b$ be a Boolean function. We can extend $b$ to a function $b^{\sim}: \overline{\mathbb{R}}^{n} \rightarrow \overline{\mathbb{R}}$ in the following way:

$$
b^{\sim}\left(t_{1}, t_{2}, \ldots, t_{n}\right)=\sup \left\{t \in \overline{\mathbb{R}} \mid b\left(\left[t_{1} \geq t\right], \ldots,\left[t_{n} \geq t\right]\right)=1\right\},
$$

where $\left[t_{1} \geq t\right]$ is a Boolean expression which equals 1 if $t_{1} \geq t$ and 0 otherwise. It is easy to show that this procedure satisfies the following properties:

$$
\left(b_{1} \cdot b_{2}\right)^{\tilde{z}}=\tilde{b_{1}} \wedge \tilde{b_{2}}, \quad\left(b_{1}+b_{2}\right)^{-}=\tilde{b_{1}} \vee \tilde{b_{2}},
$$

where $\cdot$ and + denote the logic AND and OR, respectively. For example, if $b\left(x_{1}, x_{2}, x_{3}\right)=$ $x_{1} x_{2}+x_{3}$ then $b^{\sim}\left(t_{1}, t_{2}, t_{3}\right)=\left(t_{1} \wedge t_{2}\right) \vee t_{3}$. From now on we denote a positive Boolean function and its extension to $\overline{\mathbb{R}}^{n}$ with the same symbol.

Now, if $A=\left\{a_{1}, a_{2}, \ldots, a_{n}\right\}$ and $b$ is a positive Boolean function of $n$ variables, then we can define the operator $\Psi_{b}$ on Fun $(E)$ by

$$
\Psi_{b}(F)(x)=b\left(F\left(x+a_{1}\right), \ldots, F\left(x+a_{n}\right)\right) .
$$

We show that $\Psi_{b}$ coincides with the flat operator generated by $\psi_{b}$, which we denote by $\Psi$ for the moment. Combining (6.10) with the expression for $\psi_{b}$ and (6.11) we get

$$
\begin{aligned}
\Psi(F)(x) & =\bigvee\left\{t \in \overline{\mathbb{R}} \mid x \in \psi_{b}(\mathbf{X}(F, t))\right\} \\
& =\bigvee\left\{t \in \overline{\mathbb{R}} \mid b\left(\left[F\left(X+a_{1}\right) \geq t\right], \ldots,\left[F\left(x+a_{n}\right) \geq t\right]\right)=1\right\} \\
& =b\left(F\left(x+a_{1}\right), \ldots, F\left(x+a_{n}\right)\right) \\
& =\Psi_{b}(F)(x) .
\end{aligned}
$$

This proves our claim.

One can also extend the geodesic operators introduced in $\S 5$ to grey-scale functions. Such operators have been shown to be quite useful in many applications. Refer to Vincent [48] for a number of examples.

6.5. Granulometries for grey-scale functions. We conclude this section with a brief discussion on granulometries for grey-scale functions. A general account can be found in [23]. A family of openings $\left\{\alpha_{r} \mid r>0\right\}$ on Fun $(E)$ is called a granulometry if $\alpha_{s} \leq \alpha_{r}$ for $s \geq r$. It is apparent that the flat extension of a binary granulometry to Fun $(E)$ defines again a granulometry, called flat granulometry. We argue below that this class of flat granulometries is quite important. If we want to extend the notion of Minkowski granulometry there are several 
possibilities. First, we can choose between $\mathrm{H}$-openings and T-openings. And moreover, we can think of at least two different kind of scalings. The first one is the T-scaling given by

$$
(r \cdot F)(x)=r F(X / r), \quad r>0 .
$$

This scaling acts on the spatial as well as the grey-scale variable. A second type of scaling is the H-scaling,

$$
\left(r_{*} F\right)(x)=F(X / r), \quad r>0,
$$

which acts only on the spatial variable. Both scalings are depicted in Fig. 17.
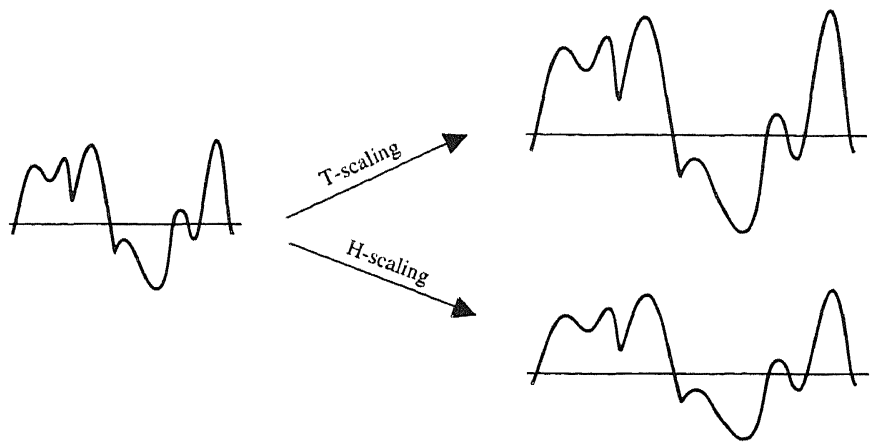

FIG. 17. T-scaling and $\mathrm{H}$-scaling of a function.

Therefore, we can distinguish at least four types of Minkowski granulometries. All of them have been discussed in more or less detail in [23]. To give the reader an impression of the underlying mathematics we consider here the so-called (T, H)-Minkowski granulometry, where the first prefix " $T$ " indicates that we consider T-openings, and the second prefix " $\mathrm{H}$ " that we consider $\mathrm{H}$-scalings. A first observation to make is that the flat extension of a Minkowski granulometry on $\mathcal{P}(E)$ is a (T, H)-Minkowski granulometry on $\operatorname{Fun}(E)$. We can formulate the following generalization of Theorem 5.5.

THEOREM 6.2. Let $\left\{\alpha_{r}\right\}$ be a $(\mathrm{T}, \mathrm{H})-$ Minkowski granulometry, then there is a family $\mathcal{G} \subseteq$ Fun $(E)$ such that

$$
\alpha_{r}(F)=\bigvee_{s \geq r} \bigvee_{G \in \mathcal{G}} F \circ s_{*} G .
$$

Conversely, if $\mathcal{G} \subseteq$ Fun $(E)$ then $\left\{\alpha_{r}\right\}$ given by (6.12) defines a $(\mathrm{T}, \mathrm{H})$-Minkowski granulometry.

For practical applications, one requires elimination of the outer supremum in (6.12) so that we end up with $\alpha_{r}(F)=\bigvee_{G \in \mathcal{G}} F \circ r G$. As in the binary case this amounts to the following condition on the structuring function $G$ :

$$
r G \circ G=r G \quad \text { for } r \geq 1 .
$$

In [23] we have proved the following theorem.

THEOREM 6.3. Let $G \in \operatorname{Fun}(E)$ be u.s.c. and have compact domain $\operatorname{dom}(G)=\{x \in$ $E \mid G(x)>-\infty\}$. Then condition (6.13) holds if and only if its domain is convex and $G$ is constant there.

In fact this result says that every structural (T, H)-Minkowski granulometry on Fun(E) ("structural" meaning that every opening involves only one structuring function) is the flat extension of a structural Minkowski granulometry on $\mathcal{P}(E)$. The proof of Theorem 6.3 employs Theorem 5.4, the Krein-Milman theorem, and Zorn's Lemma; see [23]. 
7. And so forth.... In this paper we have only been able to touch upon a few (theoretical) aspects of mathematical morphology. Many others have been kept unmentioned, and we use this last section to devote some words to two or three further issues.

We point out that the contents of this paper are largely determined by our personal interest and knowledge. This is why nothing has been said about the implementation of morphological operators. It goes without saying that this is an extremely important subject, and, for that reason it has received a lot of attention in the literature. Readers who are interested in the design of flexible data structures and fast algorithms should refer to the forthcoming book of Schmitt and Vincent [39].

Another subject which has been ignored here is the probabilistic approach. As we pointed out in the introduction, the strength of morphology lies in its intertwining with integral geometry and stochastic geometry. From the very beginning Matheron [28] and Serra [40] have emphasized the importance of a joint development of a theory of mathematical morphology and random sets. A major ingredient for such a theory of random set, as initiated by Matheron, is the so-called hit-or-miss topology on the space of closed subsets of $\mathbb{R}^{d}$. As the name suggests, this topology is closely related to the hit-or-miss operator discussed in $\S 2$. During the last ten years, the theory of morphological operators (and more particularly, morphological filters) has started to lead its own life as a tool box for image processing, including powerful algorithms for filtering, segmentation, skeletonization, etc. However, for a sound judgment of the merits of mathematical morphology as a methodology in image analysis it is essential that one bears in mind this stochastic component.

The hit-or-miss topology mentioned above has also been used to develop a theory of discretization (or digitization); see [40, Chap. VII] and [15]. Such a theory is required to bridge the gap between the analytic approach using concepts from, e.g., integral geometry, and their digital implementations. The most common discretization of an image uses the regular discrete grid, either square or hexagonal. The hexagonal grid has the advantage that it possesses more isotropy (it allows rotations over multiples of $60^{\circ}$, whereas the square grid only allows $90^{\circ}$ rotations), but the visualization of a hexagonal image requires more effort. Anyhow, discrete representations of images raise several problems which are quite familiar to people working in discrete geometry. Besides rotations, such notions as distance, convexity, homotopy, etc. also need reconsideration. But such problems concerning discrete topology and geometry cross the "morphological borders" and form a major challenge in digital image processing and computer graphics [22], [38].

For some applications, a regular grid is not the optimal discrete structure for modelling an image. For example, if $X$ is an electron microscopic image of some cell tissue it seems plausible to model the cells in this population as the vertices of a neighborhood graph. The edges of such a graph carry useful information about the spatial relationships between the individual cells. In his thesis [47] Vincent has generalized many concepts, both algebraic and geometric, from classical morphology to the graph framework. His work has been extended in [18] and [21].

We hope that we have succeeded in giving the reader a first impression of the underlying rinciples of mathematical morphology and in convincing him or her that this theory benefits om the power of a mathematical framework and poses many challenging questions.

\section{REFERENCES}

] G. J. F. BANON AND J. BARRERA, Minimal representation for translation-invariant set mappings by mathematical morphology, SIAM J. Appl. Math., 51 (1991), pp. 1782-1798.

2] G. BIRKHOFF, Lattice Theory, American Mathematical Society Colloquium Publications. Vol. 25, 3rd ed., American Mathematical Society, Providence, RI, 1967. 
[3] G. Borgefors, Distance transformations in arbitrary dimensions, Computer Vision, Graphics and Image Processing, 27 (1984), pp. 321-345.

[4] - Distance transformations in digital images, Computer Vision, Graphics and Image Processing, 34 (1986), pp. 344-371.

[5] H. Busemann, The Geometry of Geodesics, Academic Press, New York, 1955.

[6] E. R. Dougherty and C. R. Giardina, Image Processing-Continuous to Discrete, Prentice-Hall, Englewood Cliffs, NJ, 1987.

[7] C. R. Giardina AND E. R. Dougherty, Morphological Methods in Image and Signal Processing, Prentice-Hall, Englewood Cliffs, NJ, 1988.

[8] J. Goutsias, H. Heijmans, AND K. Sivakumar, Morphological operators for image sequences, Computer Vision, Graphics and Image Processing: Image Understanding, to appear.

[9] H. HADWIGER, Vorlesungen über Inhalt, Oberfläche und Isoperimetrie, Springer-Verlag, Berlin, 1957.

[10] R. M. HARALICK, S. R. STERnBERG, AND X. ZhUANG, Image analysis using mathematical morphology, IEEE Trans. Pattern Anal. Machine Intelligence, 9 (1987), pp. 532-550.

[11] M. A. HARRISON, Introduction to Switching and Automata Theory, McGraw-Hill. New York, 1965.

[12] H. J. A. M. HejJmans, Iteration of morphological transformations. CWI Quarterly, 2 (1989), pp. 19-36.

[13] — Morphological filtering and iteration, in Visual Communications and Image Processing ' 90 . Vol. 1360 of SPIE Proceedings, Lausanne, Switzerland, 1990, pp. 166-175.

[14] - Theoretical aspects of gray-level morphology, IEEE Trans. Pattern Anal. Machine Intelligence, 13 (1991), pp. 568-582.

[15] - Discretization of morphological operators, J. Visual Communication and Image Representation. 3(1992), pp. 182-193.

[16] - A note on the umbra transform in gray-scale morphology, Pattern Recognition Lett., 14 (1993), pp. $877-881$.

[17] - Morphological Image Operators, Academic Press, Boston, 1994.

[18] H. J. A. M. HeIJMANS, P. NACKEN, A. TOET, AND L. VINCENT, Graph morphology, J. Visual Communication and Image Representation, 3 (1992), pp. 24-38.

[19] H. J. A. M. HEIJMANS AND C. RONSE, The algebraic basis of mathematical morphology-part $\mathrm{Y}$ : Dilations and erosions, Computer Vision, Graphics and Image Processing, 50 (1990), pp. 245-295.

[20] H. J. A. M. Heimans and J. SeRra, Convergence, continuity and iteration in mathematical morphology, J. Visual Communication and Image Representation, 3 (1992), pp. 84-102.

[21] H. J. A. M. HeImANS AND L. VINCENT, Graph morphology in image analysis, in Mathematical Morphology in Image Processing, E. R. Dougherty, ed., Marcel Dekker, New York, 1993, Ch. 6, pp. 171-203.

[22] V. A. Kovalevsky, Finite topology as applied to image analysis, Computer Vision. Graphics and Image Processing, 46 (1989), pp. 141-161.

[23] E. J. KRAus, H. J. A. M. Heismans, AND E. R. Dougherty, Gray-scale granulometries compatible with spatial scalings, Signal Processing, 34 (1993), pp. 1-17.

[24] C. LantuéJoul and F. Maisonneuve, Geodesic methods in quantitative image analysis, Pattern Recognition, 17 (1984), pp. 177-187.

[25] F. MaisonneuvE, Ordinaux transfinis et sur- (ou sous-) potentes. Tech. Rep. N780, Ecole Nationale Supérieure des Mines de Paris, Fountainebleau, 1982

[26] P. MARAGOS, A representation theory for morphological image and signal processing, IEEE Trans. Pattern Anal. Machine Intelligence, 11 (1989), pp. 586-599.

[27] P. MARAgos AND R. W. SChafer, Morphological filters-part I: Their set-theoretic analysis and relations to linear shift-invariant filters, IEEE Trans. Acoust. Speech Signal Process., 35 (1987), pp. 1153-1169.

[28] G. MATHERON, Random Sets and Integral Geometry, John Wiley, New York, 1975.

[29] S. MUROGA, Threshold Logic and Its Applications, Wiley-Interscience, New York, 1971.

[30] P. NACKEN, Efficient algorithms for mathematical morphology based on chamfer distances, in Mathematical Morphology and its Applications to Signal Processing, J. Serra and P. Salembier, eds.. Proc. Internat. Workshop, 1993, Barcelona, Spain.

[31] - Chamfer metrics in mathematical morphology, J. Math. Imaging and Vision, 4 (1994), pp. 233-253.

[32] W. Rinow, Die Innere Geometrie der Metrischen Räume. Springer-Verlag, Berlin, 1961.

[33] J. B. T. M. RoERDINK, Mathematical morphology with non-commutatitive symmetry groups, in Mathematical Morphology in Image Processing, E. R. Dougherty, ed., Marcel Dekker. New York, 1993, Ch. 7, pp. 205-254.

[34] - On the construction of translation and rotation invariant morphological operators, in Mathematical Morphology: Theory and Hardware, R. M. Haralick, ed., Oxford University Press, to appear.

[35] C. Ronse, Lattices of inf-overfilters, Working Document WD53, Philips Research Laboratory, Brussels, Belgium, 1989. 
[36] C. Ronse, Openings: Main properties, and how to construct them, in Mathematical Morphology: Theory and Hardware, R. M. Haralick, ed., Oxford University Press, to appear.

[37] C. Ronse AND H. J. A. M. HeIJMANS, The algebraic basis of mathematical morphology-part II: Openings and closings, Computer Vision, Graphics and Image Processing: Image Understanding, 54 (1991), pp. 74-97.

[38] A. RosenFeld AND R. A. MelTeR, Digital geometry, The Mathematical Intelligencer, 11 (1989), pp. 69-72.

[39] M. SCHMITT AND L. VINCENT, Morphology: Algorithms and Applications, Cambridge University Press, to appear.

[40] J. SERRA, Image Analysis and Mathematical Morphology, Academic Press, London, 1982.

[41] - ED., Image Analysis and Mathematical Morphology. II: Theoretical Advances, Academic Press, London. 1988.

[42] J. SERRA AND L. VINCENT, An overview of morphological filtering, Circuits Systems Signal Process., 11 (1992), pp. 47-108.

[43] S. R. SteRnBerg, Grayscale morphology, Computer Vision, Graphics and Image Processing, 35 (1986), pp. 333-355.

[44] F. A. Valentine, Convex Sets, McGraw-Hill. New York, 1964.

[45] R. van Den BoomgaARd, Mathematical Morphology: Extensions towards Computer Vision, Ph.D. thesis. University of Amsterdam, 1992.

[46] - Decomposition of structuring elements, IEEE Trans. Pattern Anal. Machine Intelligence, to appear.

[47] L. VINCENT, Algorithmes Morphologiques a'Base de Files d'Attente et de Lacets. Extension aux Graphes, Ph.D. thesis, Ecole Nationale Supérieure des Mines de Paris, Fountainbleau, 1990.

[48] Morphological grayscale reconstruction in image analysis: Applications and efficient algorithms, IEEE Trans. Image Process., 2 (1993), pp. 176-201.

[49] J. XU, Decomposition of convex polygonal morphological structuring elements into neighbourhood subsets, IEEE Trans. Pattern Anal. Machine Intelligence. 13 (1991), pp. 153-162.

[50] X. ZhUANG AND R. M. HARALICK, Morphological structuring element decomposition, Computer Vision, Graphics and Image Processing, 35 (1986), pp. 370-382. 